\title{
$\Lambda$-adic modular symbols over totally real fields
}

\author{
Baskar Balasubramanyam and Matteo Longo
}

\begin{abstract}
We consider a Hida family of nearly ordinary cusp forms on a quaternion algebra defined over a totally real number field. The aim of this work is to construct a cohomology class with coefficients in a $p$-adic sheaf over an Iwasawa algebra that can be specialized to cohomology classes attached to classical cusp forms in the given Hida family. Our result extends the work of Greenberg and Stevens on modular symbols attached to ordinary Hida families when the ground field is the field of rational numbers.
\end{abstract}

Mathematics Subject Classification (2010). 11G18, 11F41, 11F67.

Keywords. Shimura varieties, Hilbert modular forms, modular symbols, Hida families.

\section{Introduction}

Let $F / \mathbb{Q}$ be a totally real field of degree $d$. We will always assume in this paper that $d>1$ because the results that we will present are generalizations of results already known ([4]) when the base field is the field of rational numbers. Denote by $r$ the ring of integers of $F$. For any abelian group $A$, define $\widehat{A}:=A \otimes_{\mathbb{Z}} \widehat{\mathbb{Z}}$, where $\widehat{\mathbb{Z}}$ is the profinite completion of $\mathbb{Z}$. For any quaternion algebra $\boldsymbol{B} / F$ let $\boldsymbol{G}=\boldsymbol{G}$ be the linear algebraic group over $\mathbb{Q}$ such that $\boldsymbol{G}(\mathbb{Q})=\boldsymbol{B}^{\times}$. Let $\boldsymbol{G}_{\mathbb{A}}$ denote the adelization of $\boldsymbol{G}$ and denote respectively by $\boldsymbol{G}_{f}, \boldsymbol{G}_{\infty}$ and $\boldsymbol{G}_{\infty}+$ the finite part, the infinite part and the connected component of $\boldsymbol{G}_{\infty}$ containing the identity. Fix a compact open subgroup $S \subseteq \boldsymbol{G}_{f}$ such that $U_{0}(\mathfrak{n}) \supseteq S \supseteq U_{1}(\mathfrak{n})$ for some integral ideal $\mathfrak{n}$ of $F$, where $U_{0}(\mathfrak{n})$ and $U_{1}(\mathfrak{n})$ are congruence groups defined in $\S 2.1$. Let $p$ be a rational prime which is prime to $2 \mathfrak{n}$ and define $S\left(p^{\alpha}\right):=S \cap U\left(p^{\alpha}\right)$ for any non-negative integer $\alpha$, where $U\left(p^{\alpha}\right)$ is also defined in $\$ 2.1$. Fix an embedding $\iota: \overline{\mathbb{Q}} \hookrightarrow \overline{\mathbb{Q}}_{p}$ and a finite extension $K$ of $\mathbb{Q}_{p}$ containing $\iota \circ \mu(F)$ for all archimedean places $\mu$ of $F$. Denote by $\mathcal{O}$ the ring of integers of $K$.

Let $I$ denote the set of embeddings of $F$ into $\mathbb{C}$. Let $n, v \in \mathbb{Z}[I]$ be fixed weight vectors such that $n+2 v \equiv 0 \bmod \mathbb{Z} t$, where $t=(1, \ldots, 1) \in \mathbb{Z}[I]$ and let $k:=n+2 t$ and $w:=v+k-t$. Following [7], we denote by $h_{k, w}^{\text {n,ord }}\left(S\left(p^{\alpha}\right), \mathcal{O}\right)$ the Hecke algebra over $\mathcal{O}$ for the space of $p$-nearly ordinary Hilbert cusp forms of 
weight $(k, w)$ and level $S\left(p^{\alpha}\right)$. In $\S 2.3$ we recall Hida's construction of the universal $p$-odinary Hecke algebra

$$
\mathcal{R}:=\overleftarrow{\alpha}_{\alpha}^{\lim } h_{2 t, t}^{\mathrm{n}, \text { ord }}\left(S\left(p^{\alpha}\right), \mathcal{O}\right)
$$

This Hecke algebra is universal in the sense that each nearly ordinary Hecke algebra $h_{k, w}^{\text {n,ord }}\left(S_{0}\left(p^{\alpha}\right), \epsilon, K\right)$ acting on the $K$-vector space of cusp forms of weight $(k, w)$, level $S_{0}\left(p^{\alpha}\right)$ and finite-order character $\epsilon: S_{0}\left(p^{\alpha}\right) / S_{1}\left(p^{\alpha}\right) \rightarrow \mathbb{C}$ is isomorphic to a residue algebra of $\mathcal{R}$. Here $S_{0}\left(p^{\alpha}\right):=S \cap U_{0}\left(p^{\alpha}\right)$ and $S_{1}\left(p^{\alpha}\right):=S \cap U_{1}\left(p^{\alpha}\right)$. More precisely, let

$$
G:={\underset{\leftarrow}{\alpha}}_{\lim _{0}} S_{0}\left(p^{\alpha}\right) \mathrm{r}^{\times} / S\left(p^{\alpha}\right) \mathrm{r}^{\times}
$$

and denote by $W$ the free part of $G$. Then $\mathcal{R}$ has a natural structure of $\tilde{\Lambda}$-algebra, where $\tilde{\Lambda}:=\mathcal{O}[[G]]$ is the Iwasawa algebra of $G$ and there are isomorphisms

$$
\mathcal{R}_{P} / P \mathcal{R}_{P} \stackrel{\sim}{\longrightarrow} h_{k, w}^{\mathrm{n}, \text { ord }}\left(S_{0}\left(p^{\alpha}\right), \epsilon, K\right)
$$

for suitable ideals $P$ of $\tilde{\Lambda}$, where $\mathcal{R}_{P}$ is the localization of $\mathcal{R}$ at $P$. See [7], Theorem 2.4 , or $\S 2.3$ for details.

Let $\Lambda:=\mathcal{O}[[W]]$ be the Iwasawa algebra of the free part $W$ of $G$. Denote by $\mathscr{L}:=\operatorname{Frac}(\Lambda)$ the field of fractions of $\mathscr{L}$ and by $\overline{\mathscr{L}}$ its algebraic closure. Fix a point

$$
\theta \in \operatorname{Hom}_{\text {cont }}^{\mathcal{O} \text {-algebras }}(\mathcal{R}, \overline{\mathscr{L}}) \text {. }
$$

By [7], Theorem 2.4, the image of $\theta$ is contained in a finite extension $\mathcal{K}$ of $\mathscr{L}$. Let $\mathcal{I}$ denote the integral closure of $\Lambda$ in $\mathcal{K}$. Say that a point

$$
\kappa \in \mathcal{X}(\mathcal{I}):=\operatorname{Hom}_{\text {cont }}^{\mathcal{O}-\text { algebras }}\left(\mathcal{I}, \overline{\mathbb{Q}}_{p}\right)
$$

is arithmetic if its restriction to $G$ has kernel equal to $P$ for some of the ideals $P$ appearing in (1). See [7], p. 150-152, or $\$ 2.3$ for a more precise notion of arithmetic point. A corollary of Theorem 2.4 in [7] states that points in $\mathcal{X}(\mathcal{I})$ correspond to $p$-adic Hilbert modular forms and arithmetic points $\kappa$ corresponds to classical $p$ nearly ordinary Hilbert modular forms $f_{\kappa}$ of suitable weight $(k, w)$, level $S_{0}\left(p^{\alpha}\right)$ and character $\epsilon$. See [7], Corollary 2.5, for details. In particular, to any arithmetic point $\kappa$ is associated a weight $(n, v)($ or $(k, w))$, a level $S_{0}\left(p^{\alpha}\right)$ and a character $\epsilon$.

Remark 1.1. Note that the Iwasawa algebra $\Lambda:=\mathcal{O}[[W]]$ of $W$ is isomorphic to the formal power series ring in $s$ variables $\mathcal{O} \llbracket X_{1}, \ldots, X_{s} \rrbracket$, where $s=d+1+\delta_{F}$ and $\delta_{F}$ is the non-negative integer appearing in Leopoldt's conjecture. An other way to state [7], Corollary 2.5, is that any $f \in S_{k, w}\left(U_{1}\left(\mathfrak{n} p^{\alpha}\right), K\right)$ has $s$-dimensional $p$-adic deformations over $\mathcal{O}$ (in the sense of [7], pp. 152-153). 
To discuss the main result of this paper, we need some technical assumptions. Assume that $\boldsymbol{B}$ is unramified at all finite primes in $F$. Denote by $F_{\mathbb{A}}$ the adele ring of $F$. By the Strong Approximation Theorem, choose $g_{\lambda} \in \mathrm{GL}_{2}\left(F_{\mathbb{A}}\right)$ for $1 \leq \lambda \leq$ $h\left(p^{\alpha}\right)$ and a suitable integer $h\left(p^{\alpha}\right)$ depending on $\alpha$ with $\left(g_{\lambda}\right)_{\mathfrak{n} p}=\left(g_{\lambda}\right)_{\infty}=1$ and such that there is the following disjoint union decomposition:

$$
\boldsymbol{G}^{B}=\coprod_{\lambda=1}^{h\left(p^{\alpha}\right)} \boldsymbol{G}_{\mathbb{Q}} g_{\lambda} S \boldsymbol{G}_{\infty^{+}} .
$$

If $\alpha=0$ write $h$ for $h(1)$. If $\boldsymbol{B}$ is indefinite, we can assume that the $g_{\lambda}$ does not depend upon $\alpha$. Define the following arithmetic groups depending on $S$ :

$$
\begin{aligned}
& \Gamma^{\lambda}\left(p^{\alpha}\right):=g_{\lambda} S\left(p^{\alpha}\right) \boldsymbol{G}_{\infty}+g_{\lambda}^{-1} \cap \boldsymbol{G}^{B}(\mathbb{Q}) \\
& \bar{\Gamma}^{\lambda}\left(p^{\alpha}\right):=\Gamma^{\lambda}\left(p^{\alpha}\right) /\left(\Gamma^{\lambda}\left(p^{\alpha}\right) \cap F^{\times}\right) .
\end{aligned}
$$

Let $I_{\boldsymbol{B}}$ be the set of infinite places where $\boldsymbol{B}$ is unramified. If $\left|I_{\boldsymbol{B}}\right|=r$, then $\boldsymbol{G}_{\infty}$ is isomorphic to $G L_{2}(\mathbb{R})^{r} \times \mathbb{H}^{d-r}$. Since $\Gamma^{\lambda}\left(p^{\alpha}\right)$ is a subgroup of $\boldsymbol{G}_{\infty}$, it is acts on $\mathfrak{S}^{r}(\mathfrak{S}$ is the complex upper half-plane).

Assumption 1.2. The groups $\bar{\Gamma}^{\lambda}:=\bar{\Gamma}^{\lambda}(1)$ are torsion free for all $\lambda=1, \ldots, h$.

See [6], Lemma 7.1, for conditions under which Assumption 1.2 is verified. In particular, there are infinitely many square-free integers for which $\bar{\Gamma}^{\lambda}\left(U_{0}(N)\right)$ is torsion-free for all $\lambda$. Under this assumption, for any $\mathcal{O}\left[\bar{\Gamma}^{\lambda}\right]$-module $E$, there is a canonical isomorphism $H^{*}\left(\Gamma^{\lambda} \backslash \mathfrak{S}^{r}, \widetilde{E}\right) \simeq H^{*}\left(\bar{\Gamma}^{\lambda}, E\right)$, where $\widetilde{E}$ is the coefficient system associated to $E$. The technical Assumption 1.2 can probably be dispensed with by employing the methods in [6], proof of Theorem 3.1, and [7], Proposition 3.1, but we keep it to enlighten the notation and the arguments. See also $\$ 7.4$ in [3] for a complete reference of this approach.

The aim of this work is to construct a cohomology class with coefficients in a $p$-adic space that can be specialized to cohomology classes attached to cusp forms in a Hida family. This is a generalization of a similar construction [4]. The main difference here is that the Iwasawa algebra $\Lambda$ in this case is isomorphic to a power series ring in at least $d+1$-variables over $\mathcal{O}$, while in the rational case it is just isomorphic to a power series ring in one variable.

Remark 1.3. In [6] Hida constructs for each weight $v$ as above an ordinary universal Hecke algebra

$$
\mathcal{R}_{v}=h_{v}^{\text {ord }}\left(U_{1}\left(p^{\infty}\right), \mathcal{O}\right)
$$

such that each $h_{k, w}^{\text {n,ord }}\left(U_{1}\left(p^{\alpha}\right), \mathcal{O}\right)$, where $k$ is parallel to $-2 v$, can be obtained as a residue algebra of $\mathcal{R}_{v}$. These Hecke algebras $\mathcal{R}_{v}$ are endowed with a structure 
of $\mathcal{O} \llbracket\left[X_{1}, \ldots, X_{\delta_{F}} \rrbracket\right.$-algebra. The Iwasawa algebra $\Lambda$ considered here has $d$ more variables in order to unify these various Hecke algebras as $v$ and the character $\epsilon$ vary.

A consequence of the fact that the Iwasawa algebra considered here is bigger than that in [4] is that the role played by the set of primitive vectors $\left(\mathbb{Z}_{p}^{2}\right)^{\prime}$ in [4] will be played in this context by the $p$-adic space

$$
X:=N C \backslash \mathrm{GL}_{2}\left(\mathrm{r}_{p}\right) \simeq \underset{\leftarrow}{\lim _{\alpha}} S\left(p^{\alpha}\right) \mathrm{r}^{\times} \backslash S \mathrm{r}^{\times}
$$

which has a greater rank. Here $N$ is the standard lower unipotent subgroup of $\mathrm{GL}_{2}\left(\mathrm{r}_{p}\right)$ and $C$ is the closure of $e:=S \cap F$ embedded diagonally in $\mathrm{GL}_{2}\left(\mathrm{r}_{p}\right)$, where $\mathrm{r}_{p}:=$ $\mathfrak{r} \otimes_{\mathbb{Z}} \mathbb{Z}_{p}$. A similar $p$-adic space has been defined and studied in [2].

Remark 1.4. In order to justify the definition of $X$, we note that, in the case of [4], there is a canonical bijection between the set of primitive vectors $\left(\mathbb{Z}_{p}^{2}\right)^{\prime}$ and the inverse limit $\lim \Gamma_{1}\left(N p^{m}\right) \backslash \Gamma_{1}(N)$, so our definition of $X$ seems to be a correct generalization of the concept of primitive vectors used in [4]. We will clarify this point of view in the following lines.

The action of the Hecke operators $U_{\mathfrak{p}}$ for prime ideals $\mathfrak{p} \mid p$ on $X$ is similar to that considered in [2]. To describe $X$ more precisely, for any prime ideal $\mathfrak{p} \mid p$ of $F$, let $\left(\mathfrak{r}_{\mathfrak{p}}^{2}\right)^{\prime}$ denote the set of primitive vectors of $\mathfrak{r}_{\mathfrak{p}}^{2}$, i.e., the set of elements $(x, y) \in \mathrm{r}_{\mathfrak{p}}^{2}$ such that at least one of $x$ and $y$ does not belong to $\mathfrak{p}$. Set $\left(\mathrm{r}_{p}^{2}\right)^{\prime}:=\prod_{\mathfrak{p} \mid p}\left(\mathrm{r}_{\mathfrak{p}}^{2}\right)^{\prime}$. Denote by $\bar{e}$ the closure of $S \cap F^{\times}$in $\mathrm{r}_{p}^{\times}$. Then $X$ can be identified via the map

$$
\gamma=\left(\begin{array}{ll}
a & b \\
c & d
\end{array}\right) \longmapsto((a, b), \operatorname{det}(\gamma))
$$

with $\bar{e} \backslash\left(\left(\mathrm{r}_{p}^{2}\right)^{\prime} \times \mathrm{r}_{p}^{\times}\right)$. Hence $X$ may be viewed as an analogue of the primitive vectors appearing in [4]. Elements of $X$ will be denoted by $((x, y), z)$.

Following [4], define $\mathbb{D}_{X}$ to be the space of $\mathcal{O}$-valued measures on $X$. This space is endowed with $\widetilde{\Lambda}$ and $\Lambda$-algebra structures. Let $X(S)$ be the Hilbert modular variety associated to $S$ and let $\mathscr{D}_{X}$ denote the local coefficient system on $X(S)$ associated to $\mathbb{D}_{X}$. We suppose from now on to the end of this section that

$$
r=\left|I_{\boldsymbol{B}}\right| \leq 1 .
$$

In particular, since $d>1, \boldsymbol{B}$ is always a division algebra. Further, since we are also assuming that $\boldsymbol{B}$ is unramified at all finite places, we have $r=0$ if $d$ is even and $r=1$ if $d$ is odd. Finally, $X(S)$ is always compact in this case. We define the space of $\Lambda$-adic modular symbols in this context to be

$$
\mathbb{W}:=H^{r}\left(X(S), D_{X}\right)=H_{\text {cpt }}^{r}\left(X(S), D_{X}\right)
$$


the $r$-th cohomology of $X(S)$ with coefficients in $\mathscr{D}_{X}$. It follows from Proposition 4.2 of [1] that this definition of $\Lambda$-adic modular symbols is consistent with the analogous definition in [4]. The group $\mathbb{W}$ is endowed with a structure of $\widetilde{\Lambda}$-module.

For any ring $R$, let $L(n, R)$ be the $R$-module of homogeneous polynomials in $2 d$ variables $X=\left(X_{\sigma}\right)_{\sigma \in I}$, and $Y=\left(Y_{\sigma}\right)_{\sigma \in I}$ of degree $n_{\sigma}$ in $X_{\sigma}, Y_{\sigma}$. Denote by $\gamma=$ $\left(\begin{array}{ll}a & b \\ c & d\end{array}\right) \mapsto \gamma^{*}:=\left(\begin{array}{cc}d & -b \\ -c & a\end{array}\right)$ the main involution of $\mathrm{M}_{2}(R)$ and define a right action of $\mathrm{GL}_{2}(R)$ on $L(n, A)$, for any $R$-algebra $A$, by $(P \mid \gamma)(X, Y):=\operatorname{det}(\gamma)^{v} P\left((X, Y) \gamma^{*}\right)$, where $(X, Y) \gamma^{*}$ is matrix multiplication. Denote by $L(n, v, A)$ the right representation of $\mathrm{GL}_{2}(R)$ thus obtained. For any character $\epsilon$ as above, write $L(n, v, \epsilon, A)$ for the $\Delta_{0}(\mathfrak{n})$-module $L(n, v, A)$ with the action of $\Delta_{0}(\mathfrak{n})$ twisted by $\epsilon$, that is, denoting by $\left.\right|_{\epsilon}$ this new action: $\left.P\right|_{\epsilon} \gamma:=\epsilon(\gamma) P \mid \gamma$ for $\gamma \in \Delta_{0}(\mathfrak{n})$.

For any arithmetic point $\kappa$ of weight $(n, v)$ and character $\epsilon$, define a specialization map $\rho_{n, v, \epsilon}: \mathbb{D}_{X} \rightarrow L(n, v, \epsilon, K)$ by

$$
\mu \longmapsto \rho_{n, v, \epsilon}(\mu):=\int_{X^{\prime}} \epsilon(x) z^{v}(x Y-y X)^{n} d \mu(x, y, z),
$$

where $X^{\prime}$ the subset of $X$ consisting of elements $((x, y), z)$ such that $x \in \mathrm{r}_{p}^{\times}$. The map $\rho_{n, v, \epsilon}$ induces a map on the cohomology groups which we again denote by the same symbol

$$
\rho_{n, v, \epsilon}: \mathbb{W} \longrightarrow H^{r}\left(X\left(S_{0}\left(p^{\alpha}\right)\right), \mathscr{L}(n, v, \epsilon, K)\right) .
$$

Here $S_{0}\left(p^{\alpha}\right)$ is the level of the modular form $f_{\kappa}$ associated to the arithmetic point $\kappa$ and $\mathscr{L}(n, v, \epsilon, K)$ is the coefficient system on $S_{0}\left(p^{\alpha}\right)$ associated to $L(n, v, \epsilon)$. By following [4] and [2], a corresponding control theorem for these specialization maps is stated in Theorem 3.7 and proved in $\$ 3.7$. More precisely, fix $\theta: \mathcal{R} \rightarrow \overline{\mathscr{L}}$ and an arithmetic $\kappa: \mathcal{I} \rightarrow \overline{\mathbb{Q}}_{p}$. Let $P_{\kappa}$ be the kernel of $\theta_{\kappa}:=\kappa \circ \theta$ and $K_{\kappa}$ the image of $\theta_{\kappa}$. Set

$$
\mathbb{W}_{\kappa}:=H^{r}\left(S_{0}\left(p^{\alpha}\right), \mathscr{L}\left(n, v, \epsilon, K_{\kappa}\right)\right) .
$$

The map $\rho_{n, v, \epsilon}$ extends to the localization $\mathcal{R}_{P_{\kappa}}$ of $\mathcal{R}$ at $P_{\kappa}$ and one obtains a map

$$
\rho_{\kappa}: \mathbb{W} \otimes_{\Lambda} \mathcal{R}_{P_{\kappa}} \longrightarrow \mathbb{W}_{\kappa}
$$

For any $\mathcal{O}[T(p)]$-module $M$, let $M^{\text {ord }}$ denote its ordinary part, that is, the maximal subspace of $M$ on which the Hecke operator $T(p)$ acts as a unit. Let $h: \mathcal{H} \rightarrow \mathcal{R}$ be the natural map obtained by the action of Hecke operators on $\Lambda$-adic cusp forms. For any arithmetic point $\kappa$, let $h_{\kappa}$ be the composition of $h$ with the localization morphism $\mathcal{R} \rightarrow \mathcal{R}_{P_{\kappa}}$. For any $\mathscr{H} \otimes_{\Lambda} \mathcal{R}_{P_{\kappa}}$-module $M$, let $M^{h_{\kappa}}$ denote the subset of $M$ consisting of those $m \in M$ such that $(T(\mathfrak{q}) \otimes 1) m=\left(1 \otimes h_{\kappa}(T(\mathfrak{q}))\right) \cdot m$ for all prime ideals $q$ in $r$. If $f_{\kappa}$ is a classical eigenform for an arithmetic point $\kappa$, let

$$
\mathbb{W}_{\kappa}^{f_{\kappa}}=\left\{\phi \in \mathbb{W}_{\kappa} \mid T_{0}(\mathfrak{q}) \phi=a_{\mathfrak{q}}(g) \phi\right\}
$$


denote the $f_{\kappa}$-eigenspace of $\mathbb{W}_{\kappa}$, where $a_{\mathfrak{q}}(g)$ is the eigenvalue of the $T_{0}(\mathfrak{q})$ operator on $f_{\kappa}$. Hence, $\rho_{\kappa}$ induces a map

$$
\rho_{\kappa}:\left(\mathbb{W} \otimes_{\Lambda} \mathcal{R}_{P_{\kappa}}\right)^{h_{\kappa}} \longrightarrow \mathbb{W}_{\kappa}^{f_{\kappa}} .
$$

Theorem 1.5. Fix $\theta: \mathcal{R} \rightarrow \mathcal{I}$ and let $\kappa: \mathcal{I} \rightarrow \overline{\mathbb{Q}}_{p}$ be an arithmetic point of weight $\left(n_{\kappa}, v_{\kappa}\right)$ and character $\epsilon_{\kappa}$. Assume that $\mathcal{R}_{P_{\kappa}}$ is a regular local ring. Then the map

$$
\rho_{\kappa}:\left(\mathbb{W} \otimes_{\Lambda} \mathcal{R}_{P_{\kappa}}\right)^{h_{\kappa}} / P_{\kappa}\left(\mathbb{W} \otimes_{\Lambda} \mathcal{R}_{P_{\kappa}}\right)^{h_{\kappa}} \rightarrow \mathbb{W}_{\kappa}^{f_{\kappa}}
$$

is an isomorphism.

A more general statement taking into account the action of the complex conjugation on $\mathbb{W}$ and $\mathbb{W}_{\kappa}$ when $r=1$ is given in Theorem 3.7.

Acknowledgments. This paper is part of the Ph.D thesis of the first author. The first author would like to sincerely thank his advisor Prof. F. Diamond for his help and encouragement. Both authors would like to thank Prof. H. Darmon for suggesting the problem and for his advice and support during their visits to McGill University. Finally, we sincerely thank the referee for his careful reading of the manuscript and for suggesting many valuable corrections and improvements.

\section{Hida theory for totally real fields}

2.1. Cusp forms over quaternion algebras. Let $F$ be a totally real field of degree $d>1$ over $\mathbb{Q}$ and denote by $\mathrm{r}$ its ring of integers. Denote by $F_{\mathbb{A}}$ the adele ring of $F$ and by $\widehat{F}$ the ring of finite adeles. Let $I$ denote the set of embeddings of $F$ into $\mathbb{C}$.

Fix a quaternion algebra $\boldsymbol{B} / F$ which is unramified at all finite places of $F$, let $I_{\boldsymbol{B}} \subseteq I$ be the set of archimedean places of $F$ where $\boldsymbol{B}$ is split and denote by $r=\left|I_{\boldsymbol{B}}\right| \in\{0, \ldots, d\}$ its cardinality.

Let $n, v \in \mathbb{Z}[I]$ be fixed weight vectors such that $n+2 v \equiv 0 \bmod \mathbb{Z} t$, where $t=(1, \ldots, 1) \in \mathbb{Z}[I]$ and let $k:=n+2 t$ and $w:=v+k-t$. Let $J \subset I_{\boldsymbol{B}}$ and for any $1 \leq \lambda \leq h(U)$, denote by $S_{k, w, J}\left(\Gamma^{\lambda}(U), \boldsymbol{B}, \mathbb{C}\right)$ the $\mathbb{C}$-vector space of cusp forms which are defined in [6], §2, and set

$$
S_{k, w, J}(U, \boldsymbol{B}, \mathbb{C}):=\prod_{\lambda=1}^{h(U)} S_{k, w, J}\left(\Gamma^{\lambda}(U), \boldsymbol{B}, \mathbb{C}\right) .
$$

If $\boldsymbol{B}$ and $\boldsymbol{B}^{\prime}$ are quaternion algebras over $F$ unramified at all finite places, by [6], Theorem 2.1, we know that then there exists an isomorphism

$$
i_{U}: S_{k, w, I_{B}}(U, \boldsymbol{B}, \mathbb{C}) \stackrel{\sim}{\longrightarrow} S_{k, w, I_{B^{\prime}}}\left(U, \boldsymbol{B}^{\prime}, \mathbb{C}\right) .
$$


Also by [7], Theorem 2.2, there exists an isomorphism between $S_{k, w, J}(U, \boldsymbol{B}, \mathbb{C})$ and $S_{k, w, I_{\boldsymbol{B}}}(U, \boldsymbol{B}, \mathbb{C})$ for any $J \subset I_{\boldsymbol{B}}$. These isomorphisms also commute with the Hecke operators.

Let $R$ be any maximal order in $\boldsymbol{B}$. We fix an identification $\hat{R}=R \otimes \hat{r} \cong M_{2}(\hat{r})$ where $\hat{r}=r \otimes \widehat{\mathbb{Z}}$. For any ideal $\mathfrak{m} \subseteq \mathrm{r}$ define the following open compact semigroups of $\mathrm{GL}_{2}(\hat{F})$ :

$$
\begin{gathered}
\Delta_{0}(\mathfrak{m}):=\left\{\left(\begin{array}{ll}
a & b \\
c & d
\end{array}\right) \in \mathrm{GL}_{2}(\widehat{F}) \cap \mathrm{M}_{2}(\hat{\mathfrak{r}}): c \equiv 0 \quad(\bmod \mathfrak{m})\right\}, \\
\Delta_{1}(\mathfrak{m}):=\left\{\left(\begin{array}{ll}
a & b \\
c & d
\end{array}\right) \in \Delta_{0}(\mathfrak{m}): a \equiv 1 \quad(\bmod \mathfrak{m})\right\}, \\
U_{0}(\mathfrak{m}):=\left\{\gamma \in \Delta_{0}(\mathfrak{m}): \operatorname{det}\left(\gamma_{\mathfrak{q}}\right) \in \mathfrak{r}_{\mathfrak{q}}^{\times}, \text {for all prime ideals } \mathfrak{q} \subseteq \mathfrak{r}\right\}, \\
U_{1}(\mathfrak{m}):=U_{0}(\mathfrak{m}) \cap \Delta_{1}(\mathfrak{m}), \\
U(\mathfrak{m}):=\left\{\left(\begin{array}{ll}
a & b \\
c & d
\end{array}\right) \in U_{1}(\mathfrak{m}): d_{\mathfrak{q}}-1 \in \mathfrak{m} \mathfrak{r}_{\mathfrak{q}} \text { for all prime ideals } \mathfrak{q} \mid \mathfrak{m}\right\} .
\end{gathered}
$$

Fix an ideal $\mathfrak{n} \subseteq \mathfrak{r}$, a rational prime $p$ prime to $2 \mathfrak{n}$ and an open compact subgroup $S \subseteq \mathrm{GL}_{2}(\hat{\mathfrak{r}})$ such that $U_{0}(\mathfrak{n}) \supseteq S \supseteq U_{1}(\mathfrak{n})$ and the $p$-component $S_{p}$ of $S$ is $\mathrm{GL}_{2}\left(\mathrm{r}_{p}\right)$. For any positive integer $\alpha$, set

$$
S_{0}\left(p^{\alpha}\right):=S \cap U_{0}\left(p^{\alpha}\right), \quad S_{1}\left(p^{\alpha}\right):=S \cap U_{1}\left(p^{\alpha}\right), \quad S\left(p^{\alpha}\right):=S \cap U\left(p^{\alpha}\right) .
$$

Denote by $\Gamma^{\lambda}\left(p^{\alpha}\right)$ and $\bar{\Gamma}^{\lambda}\left(p^{\alpha}\right)$ (respectively, $\Gamma_{0}^{\lambda}\left(p^{\alpha}\right)$ and $\bar{\Gamma}_{0}^{\lambda}\left(p^{\alpha}\right) ; \Gamma_{1}^{\lambda}\left(p^{\alpha}\right)$ and $\left.\bar{\Gamma}_{1}^{\lambda}\left(p^{\alpha}\right)\right)$ the arithmetic groups associated as in (2) to $S\left(p^{\alpha}\right)$ (respectively, $S_{0}\left(p^{\alpha}\right)$; $\left.S_{1}\left(p^{\alpha}\right)\right)$.

When $\boldsymbol{B}=M_{2}(F)$ we set

$$
\begin{gathered}
S_{k, w}(U, \mathbb{C}):=S_{k, w, I}\left(U, M_{2}(F), \mathbb{C}\right), \\
S_{k, w}\left(\Gamma^{\lambda}(U), \mathbb{C}\right):=S_{k, w, I}\left(\Gamma^{\lambda}(U), M_{2}(F), \mathbb{C}\right) .
\end{gathered}
$$

Any modular form $f_{\lambda} \in S_{k, w}\left(\Gamma^{\lambda}\left(p^{\alpha}\right), \mathbb{C}\right)$ has a Fourier expansion of the form

$$
f_{\lambda}(z)=\sum_{\xi \in \mathrm{g}_{\lambda} \delta, \xi \gg 0} a_{\lambda}(\xi) e^{2 \pi i(\xi \cdot z)},
$$

where the notation is as follows: $\mathfrak{g}_{\lambda}$ is an ideal represented by the norm of $g_{\lambda}$, $\delta$ is the different ideal of $F / \mathbb{Q}, \xi \gg 0$ if and only if, by definition, $\xi$ is totally positive, and $(\xi \cdot z):=\sum_{\sigma \in I} \sigma(\xi) z_{\sigma}$ is the scalar product. For details, see [6], Corollary 4.3.

2.2. Hecke operators. The right action on $S_{k, w}\left(S\left(p^{\alpha}\right), \mathbb{C}\right)$ of the Hecke algebra $R\left(S\left(p^{\alpha}\right), \Delta_{0}\left(\mathfrak{n} p^{\alpha}\right)\right)$, which is by definition the free $\mathbb{Z}$-module generated by double cosets

$$
T(x):=S\left(p^{\alpha}\right) x S\left(p^{\alpha}\right)
$$


for $x \in \Delta_{0}\left(\mathfrak{n} p^{\alpha}\right)$ with multiplication defined by

$$
\sum_{i} a_{i} T(x) \cdot \sum_{j} b_{j} T(y):=\sum_{i, j} a_{i} b_{j} T\left(x_{i} y_{j}\right),
$$

can be described as follows. Fix $x \in \Delta_{0}\left(\mathfrak{n} p^{\alpha}\right)$ and $1 \leq \lambda \leq h$. Let $\mu \in\{1, \ldots, h\}$ be such that $\operatorname{det}(x) t_{\lambda} t_{\mu}^{-1}$ is trivial in the strict class group of $F$. Set $x_{\lambda}:=\left(\begin{array}{ll}1 & 0 \\ 0 & t_{\lambda}\end{array}\right)$. Let $\alpha_{\lambda}$ be such that $S\left(p^{\alpha}\right) x S\left(p^{\alpha}\right)=S\left(p^{\alpha}\right) x_{\lambda}^{-1} \alpha_{\lambda} x_{\mu} S\left(p^{\alpha}\right)$ and form the finite disjoint coset decomposition $\Gamma_{\lambda}\left(p^{\alpha}\right) \alpha_{\lambda} \Gamma_{\mu}\left(p^{\alpha}\right)=\sum_{j} \Gamma_{\lambda}\left(p^{\alpha}\right) \alpha_{\lambda, j}$, where $\alpha_{\lambda, j} \in$ $\mathrm{GL}_{2}(F) \cap x_{\lambda} \Delta_{0}\left(\mathfrak{n} p^{\alpha}\right) x_{\mu}^{-1}$. Define

$$
g_{\mu}:=\sum_{j} f_{\lambda} \mid \alpha_{\lambda, j} \quad \text { and } \quad f \mid T(x):=\left(g_{1}, \ldots, g_{h}\right) .
$$

Denote by $\widetilde{F}$ the composite field of all the images $\sigma(F)$ of $F$ under the elements $\sigma \in I$ and by $\tilde{\mathfrak{r}}$ its ring of integers. Fix an $\tilde{\mathfrak{r}}$-algebra $A \subseteq \mathbb{C}$ such that for every $x \in \widehat{F}^{\times}$and every $\sigma \in I$, the $A$-ideal $x^{\sigma} A$ is generated by a single element of $A$. For any prime ideal $\mathfrak{q} \subseteq \mathfrak{r}$, choose a generator $\left\{\mathfrak{q}^{\sigma}\right\} \in A$ of the principal ideal $\mathfrak{q}^{\sigma} A$. Define $\left.\{\mathfrak{q}\}^{v}:=\prod_{\sigma \in I}\{\mathfrak{q}\}^{\sigma}\right\}^{v_{\sigma}}$. Write a fractional ideal $\mathfrak{m}$ of $\mathfrak{r}$ as a product of prime ideals $\mathfrak{m}=\prod_{\mathfrak{q}} \mathfrak{q}^{m(\mathfrak{q})}$ and define $\{\mathfrak{m}\}^{v}:=\prod_{\mathfrak{q}}\left(\{\mathfrak{q}\}^{v}\right)^{m(\mathfrak{q})}$. For any element $x \in \hat{F}^{\times}$, denote by $\mathfrak{m}_{x}$ the fractional $\mathfrak{r}$-ideal corresponding to $x$ and define $\{x\}^{v}:=\left\{\mathfrak{m}_{x}\right\}^{v}$. Modify the Hecke operators $T(x) \in R\left(S\left(p^{\alpha}\right), \Delta_{0}\left(\mathfrak{n} p^{\alpha}\right)\right)$ by setting

$$
T_{0}(x):=\left(\{\operatorname{det}(x)\}^{v}\right)^{-1} T(x) .
$$

Denote by $h_{k, w}\left(S\left(p^{\alpha}\right), A\right)$ the $A$-subalgebra of $\operatorname{End}\left(S_{k, w}\left(S\left(p^{\alpha}\right), \mathbb{C}\right)\right)$ generated over $A$ by operators $T_{0}(x)$ for $x \in \Delta_{0}\left(\mathfrak{n} p^{\alpha}\right)$. By [7], Proposition 1.1, $h_{k, w}\left(S\left(p^{\alpha}\right), A\right)$ is commutative.

For any $x \in F$ and $m=\left(m_{\sigma}\right)_{\sigma} \in \mathbb{Z}[I]$, set $x^{m}:=\prod_{\sigma \in I} \sigma(x)^{m_{\sigma}}$. For any integral ideal $\mathfrak{m}$, choose $\lambda=\lambda(\mathfrak{m})$ such that $\mathfrak{m}$ is equivalent to $t_{\lambda} \downarrow$ in the strict ideal class group of $F$ and let $\xi_{\mathfrak{m}} \in \mathrm{t}_{\lambda} \delta$ be such that $\xi_{\mathfrak{m}} \gg 0$ and $\mathfrak{m}=\xi_{\mathfrak{m}}\left(\mathrm{t}_{\lambda} \delta\right)^{-1}$. Define the modified Fourier coefficients as in [6], Corollary 3.4:

$$
C(\mathfrak{m}, f):=\frac{a_{\lambda}\left(\xi_{\mathfrak{m}}\right) \xi_{\mathfrak{m}}^{v}}{b_{v, \lambda}}, \quad \text { where } b_{v, \lambda}:=\frac{\mathrm{N}\left(\mathrm{t}_{\lambda}\right)}{\left\{\left(\mathrm{t}_{\lambda} \delta\right)^{v}\right\}}
$$

Now suppose that $f \in S_{k, w}\left(S\left(p^{\alpha}\right), \mathbb{C}\right)$ is an eigenform for $h\left(S\left(p^{\alpha}\right), A\right)$ such that $C\left(\mathrm{r}, f_{\lambda}\right)=1$ for all $\lambda=1, \ldots, h$ (call such a form normalized). Then by [6], Corollary 4.2,

$$
f \mid T(\mathfrak{m})=C(\mathfrak{m}, f) f .
$$

The group $G_{\alpha}:=S_{0}\left(p^{\alpha}\right) \mathrm{r}^{\times} / S\left(p^{\alpha}\right) \mathrm{r}^{\times}$acts on $S_{k, w}\left(S\left(p^{\alpha}\right), \mathbb{C}\right)$ via the operator $\omega\left(a_{p}^{n+2 v}\right)^{-1} T(x)$ for $x=\left(\begin{array}{ll}a & b \\ c & d\end{array}\right) \in S_{0}\left(p^{\alpha}\right)$, where $\omega$ is the Teichmüller character. 
For any prime ideal $q \subseteq \mathfrak{r}$, choose an element $q \in \hat{\mathfrak{r}}^{\times}$such that $q \hat{\mathfrak{r}}=\hat{\mathfrak{q}}$ and the $\mathfrak{l}$-component $q_{\mathfrak{r}}$ of $q$ is equal to 1 for all prime ideals $\mathfrak{l} \subseteq \mathfrak{r}, \mathfrak{l} \neq \mathfrak{q}$. Define $T(\mathfrak{q}):=T\left(\begin{array}{ll}1 & 0 \\ 0 & q\end{array}\right)$ for all prime ideals $\mathfrak{q} \subseteq \mathrm{r}$ and $T(\mathfrak{q}, \mathfrak{q}):=T\left(\begin{array}{ll}q & 0 \\ 0 & q\end{array}\right)$ for prime ideals $\mathfrak{q} \subseteq \mathfrak{r}$ such that $\mathfrak{q} \nmid \mathfrak{n}$. By [7], Proposition 1.1, if $S \supseteq U_{1}(\mathfrak{n})$, then $h_{k, w}\left(S\left(p^{\alpha}\right), A\right)$ is generated over $A$ by the operators induced from the action of $G_{\alpha}$ and $T_{0}(\mathfrak{q})$ for all prime ideals $\mathfrak{q}$.

If $f$ is an eigenform for the Hecke algebra $h_{k, w}\left(S\left(p^{\alpha}\right), A\right)$, then its eigenvalues are algebraic numbers. If $k \sim t$, then they are algebraic integers. Define $S_{k, w}\left(S\left(p^{\alpha}\right), A\right) \subseteq S_{k, w}\left(S\left(p^{\alpha}\right), \mathbb{C}\right)$ to be the $A$-module consisting of forms whose Fourier expansion has coefficients in $A$. The $A$-module $S_{k, w}\left(S\left(p^{\alpha}\right), A\right)$ is stable under the action of $h_{k, w}\left(S\left(p^{\alpha}\right), A\right)$.

2.3. Nearly ordinary Hecke algebras. Choose an embedding $\iota: \overline{\mathbb{Q}} \hookrightarrow \overline{\mathbb{Q}}_{p}$, so that any algebraic number is equipped with a $p$-adic valuation. Fix a ring of integers $\mathcal{O}$ of a finite extension of the completion of $\iota(\tilde{F})$. After choosing an embedding $i: \overline{\mathbb{Q}}_{p} \hookrightarrow \mathbb{C}$, the $\tilde{\mathfrak{r}}$-algebra $\mathcal{O}$ satisfies the conditions of $\$ 2.2$.

By Lemma 2.2 of [7], $h_{k, w}\left(S\left(p^{\alpha}\right), \mathcal{O}\right)$ is free of finite rank over $\mathcal{O}$ and hence can be decomposed as a direct sum

$$
h_{k, w}\left(S\left(p^{\alpha}\right), \mathcal{O}\right)=h_{k, w}^{\mathrm{n}, \text { ord }}\left(S\left(p^{\alpha}\right), \mathcal{O}\right) \oplus h_{k, w}^{\mathrm{ss}}\left(S\left(p^{\alpha}\right), \mathcal{O}\right)
$$

such that the image of $T_{0}(p)$ in the first factor, the nearly ordinary part, is a unit while its image in the other factor is topologically nilpotent. For any pair of non-negative integers $\beta \geq \alpha$, the map $T_{0}(x) \mapsto T_{0}(x)$ for $x \in \Delta_{0}\left(\mathfrak{n} p^{\alpha}\right)$ induces a surjective ring homomorphism $\rho_{\alpha}^{\beta}: h_{k, w}\left(S\left(p^{\beta}\right), \mathcal{O}\right) \rightarrow h_{k, w}\left(S\left(p^{\alpha}\right), \mathcal{O}\right)$. Define

and

$$
h_{k, w}\left(S\left(p^{\infty}\right), \mathcal{O}\right):=\lim _{\longleftarrow} h_{k, w}\left(S\left(p^{\alpha}\right), \mathcal{O}\right)
$$

$$
h_{k, w}^{\mathrm{n}, \text { ord }}\left(S\left(p^{\infty}\right), \mathcal{O}\right):=\lim _{\longleftarrow} h_{k, w}^{\mathrm{n}, \text { ord }}\left(S\left(p^{\alpha}\right), \mathcal{O}\right),
$$

where the inverse limits are with respect to the maps $\rho_{\alpha}^{\beta}$. By [7], Theorem 2.3, for any weight $(k, w)$ there is an isomorphism $h_{k, w}\left(S\left(p^{\infty}\right), \mathcal{O}\right) \simeq h_{2 t, t}\left(S\left(p^{\infty}\right), \mathcal{O}\right)$ which takes $T(\mathfrak{q})$ to $T(\mathfrak{q})$ and $T(\mathfrak{q}, \mathfrak{q})$ to $T(\mathfrak{q}, \mathfrak{q})$ for all prime ideals $\mathfrak{q} \nmid p$. This isomorphism induces an isomorphism between the nearly ordinary parts

$$
h_{k, w}^{\mathrm{n}, \text { ord }}\left(S\left(p^{\infty}\right), \mathcal{O}\right) \simeq \mathcal{R}:=h_{2 t, t}^{\mathrm{n}, \text { ord }}\left(S\left(p^{\infty}\right), \mathcal{O}\right) .
$$

Set $S_{F}:=S \cap \hat{F}^{\times}, S_{F}\left(p^{\alpha}\right):=S\left(p^{\alpha}\right) \cap \hat{F}^{\times}, \bar{Z}_{\alpha}:=S_{F} \mathrm{r}^{\times} / S_{F}\left(p^{\alpha}\right) \mathfrak{r}^{\times}$and $\bar{Z}_{\infty}:=\lim _{\alpha} \bar{Z}_{\alpha}$. By [7], Lemma 2.1, the map $\left(\begin{array}{ll}a & b \\ c & d\end{array}\right) \mapsto\left(a_{p}^{-1} d_{p}, a\right)$ induces an isomorphism $G_{\alpha} \simeq\left(\mathrm{r} / p^{\alpha}\right)^{\times} \times \bar{Z}_{\alpha}$ and hence an isomorphism

$$
G:=\lim _{\longleftarrow} G_{\alpha} \simeq \mathrm{r}_{p}^{\times} \times \bar{Z}_{\infty}
$$


Since $h_{k, w}\left(S\left(p^{\alpha}\right), \mathcal{O}\right)$ is an $\mathcal{O}\left[G_{\alpha}\right]$-algebra and this algebra structure is compatible with the maps $\rho_{\alpha}^{\beta}$, it follows that $h_{k, w}^{\text {n,ord }}\left(S\left(p^{\infty}\right), \mathcal{O}\right)$ is a $\widetilde{\Lambda}:=\mathcal{O}[[G]]$-algebra. Write $G \simeq W \times G^{\text {tors }}$ where $G^{\text {tors }}$ is the torsion subgroup of $G$ and $W$ is torsion-free. Note that $W$ is well determined only up to isomorphism; fix from now on a choice of $W$. The ring $\Lambda:=\mathcal{O}[[W]]$ is isomorphic to the power series ring $\mathcal{O}\left[\left[X_{1}, \ldots, X_{s}\right]\right.$ in $d<s<2 d$ variables. If Leopoldt's conjecture holds, then $s=d+1$. By [7], Theorem 2.4 , the nearly ordinary Hecke algebra $\mathcal{R}=h_{2 t, t}^{\mathrm{n}, \mathrm{rd}}\left(S\left(p^{\infty}\right), \mathcal{O}\right)$ is a torsion-free $\Lambda$-module of finite type.

For any finite-order character $\epsilon: S_{0}\left(p^{\alpha}\right) / S_{1}\left(p^{\alpha}\right) \rightarrow \overline{\mathbb{Q}}^{\times}$, define characters $\epsilon_{\lambda}: \Gamma_{0}^{\lambda}\left(p^{\alpha}\right) / \Gamma_{1}^{\lambda}\left(p^{\alpha}\right) \rightarrow \mathbb{C}^{\times}$by setting $\epsilon_{\lambda}(\gamma):=\epsilon\left(t_{\lambda}^{-1} \gamma t_{\lambda}\right)$. Denote by

$$
S_{k, w}\left(S_{0}\left(p^{\alpha}\right), \epsilon, \mathbb{C}\right)
$$

the $\mathbb{C}$-subspace of $S_{k, w}\left(S\left(p^{\alpha}\right), \mathbb{C}\right)$ consisting of forms $f=\left(f_{1}, \ldots, f_{h}\right)$ such that for any $\lambda=1, \ldots, h$ and any $\gamma \in \Gamma_{0}^{\lambda}\left(p^{\alpha}\right),\left(f_{\lambda} \mid \gamma\right)(z)=\epsilon_{\lambda}^{-1}(\gamma) f(z)$. Suppose that $\mathcal{O}$ contains the values of $\epsilon$ and set $S_{k, w}\left(S_{0}\left(p^{\alpha}\right), \epsilon, \mathcal{O}\right):=S_{k, w}\left(S_{0}\left(p^{\alpha}\right), \epsilon, \mathbb{C}\right) \cap$ $S_{k, w}\left(S\left(p^{\alpha}\right), \mathcal{O}\right)$. Denote by

$$
h_{k, w}\left(S_{0}\left(p^{\alpha}\right), \epsilon, \mathcal{O}\right)
$$

the $\mathcal{O}$-subalgebra of $\operatorname{End}\left(S_{k, w}\left(S_{0}\left(p^{\alpha}\right), \epsilon, \mathbb{C}\right)\right)$ generated over $\mathcal{O}$ by operators $T_{0}(x)$ for $x \in \Delta_{0}\left(\mathfrak{n} p^{\alpha}\right)$. Finally define $h_{k, w}^{\mathrm{n}, \text { ord }}\left(S_{0}\left(p^{\alpha}\right), \epsilon, \mathcal{O}\right)$ to be the maximal factor of $h_{k, w}\left(S_{0}\left(p^{\alpha}\right), \epsilon, \mathcal{O}\right)$ such that the image of $T_{0}(p)$ is a unit in that factor. (In the following the $\epsilon_{\lambda}$ 's will often be simply denoted by $\epsilon$ ).

Definition 2.1. An eigenform $f \in S_{k, w}\left(S_{0}\left(p^{\alpha}\right), \epsilon, \mathbb{C}\right)$ for the Hecke algebra

$$
h_{k, w}\left(S\left(p^{\alpha}\right), A\right)
$$

is said to be $p$-nearly ordinary if the eigenvalue of $T_{0}(p)$ is a $p$-adic unit.

Denote by $\mathfrak{r}_{+}^{\times}$the group of totally positive units of $\mathfrak{r}$. Define

$$
Z_{\alpha}:=S_{F} \mathrm{r}_{+}^{\times} / S_{F}\left(p^{\alpha}\right) \mathrm{r}_{+}^{\times}
$$

and $Z_{\infty}:=\lim Z_{\alpha}$. The kernel of the natural surjection map $Z_{\infty} \rightarrow \bar{Z}_{\infty}$ is finite and annihilated by a power of 2 . Denote by $\chi_{\text {cyc }}: Z_{\infty} \rightarrow \mathbb{Z}_{p}^{\times}$the cyclotomic character defined by $\chi_{\text {cyc }}(x)=x^{t}=\prod_{\sigma \in I} \sigma(x)=\mathrm{N}(x)$. Let $\epsilon: Z_{\infty} \rightarrow \mathcal{O}^{\times}$be a character factoring through $Z_{\alpha}$. Suppose that $\epsilon \chi_{\text {cyc }}^{n+2 v}$ factors through $\bar{Z}_{\infty}$, where if $n+2 v=$ $m t$ with $m \in \mathbb{Z}$, then $\chi_{\mathrm{cyc}}^{n+2 v}$ is by definition $\chi_{\mathrm{cyc}}^{m}$. Let

$$
P_{n, v, \epsilon}: G \simeq \mathfrak{r}_{p}^{\times} \times \bar{Z}_{\infty} \longrightarrow \mathcal{O}^{\times}
$$


be the character defined by $P_{n, v, \epsilon}(a, z):=\epsilon \chi_{\mathrm{cyc}}^{n+2 v}(z) a^{v}$. Denote by the same symbol the homomorphism $\tilde{\Lambda} \rightarrow \mathcal{O}$ induced from $P_{n, v, \epsilon}$ by extension of scalars. The kernel of this homomorphism is a prime ideal of $\widetilde{\Lambda}$, denoted be the same symbol $P_{n, v, \epsilon}$. To simplify notation, set $P:=P_{n, v, \epsilon}$. Let $\widetilde{\Lambda}_{P}$ (respectively, $\mathcal{R}_{P}$ ) denote the localization of $\tilde{\Lambda}$ (respectively, $\mathcal{R}$ ) in $P$. Then $\mathcal{R}_{P}$ is free of finite rank over $\widetilde{\Lambda}_{P}$ and the natural surjective morphism $\mathcal{R} \rightarrow h_{k, w}\left(S_{0}\left(p^{\alpha}\right), \epsilon, \mathcal{O}\right)$ induces by [7], Theorem 2.4, an isomorphism

$$
\mathcal{R}_{P} / P \mathcal{R}_{P} \simeq h_{k, w}\left(S_{0}\left(p^{\alpha}\right), \epsilon, K\right),
$$

where $K:=\operatorname{Frac}(\mathcal{O})$ is the fraction field of $\mathcal{O}$. In particular, the dimension of the $K$-vector space $h_{k, w}\left(S_{0}\left(p^{\alpha}\right), \epsilon, K\right)$ does not depend on $\epsilon$ and $(k, w)$ and is equal to the $\Lambda_{P}$-rank of $\mathcal{R}_{P}$.

Let $\mathscr{L}:=\operatorname{Frac}(\Lambda)$ be the fraction field of $\Lambda$ and fix an algebraic closure $\overline{\mathscr{L}}$ of $\mathscr{L}$. Let $\theta: \mathcal{R} \rightarrow \overline{\mathscr{L}}$ be a $\Lambda$-algebra homomorphism. The image $\operatorname{Im}(\theta)$ of $\theta$ is finite over $\Lambda$. Denote by $\mathcal{I}$ the integral closure of $\operatorname{Im}(\theta)$ in the fraction field $\mathcal{K}:=\operatorname{Frac}(\operatorname{Im}(\theta))$. Define

$$
\mathcal{X}(\mathcal{I}):=\operatorname{Hom}_{\mathcal{O}-\mathrm{alg}}\left(\mathcal{I}, \overline{\mathbb{Q}}_{p}\right)
$$

and denote by $\mathcal{A}(\mathfrak{I})$ the subset of $\kappa \in \mathcal{X}(\mathfrak{I})$ consisting of points whose restriction to $\Lambda$ coincides with the restriction to $\Lambda$ of some character $P_{n(\kappa), v(\kappa), \epsilon(\kappa)}$ as above. Points in $\mathcal{A}(\mathcal{I})$ are called arithmetic. In this case, set $k(\kappa):=n(\kappa)-2 t$ and $w(\kappa):=k(\kappa)+v(\kappa)-t$. Let $C(\kappa)$ denote the conductor of $\epsilon$ restricted to the torsion free part $W$ of $\bar{Z}_{\infty}$ and $\epsilon_{W}$ the restriction of $\epsilon$ to $W$. Let $\bar{Z}_{\infty}^{\text {tors }}$ denote the maximal torsion subgroup of $\bar{Z}_{\infty}$ and let $\psi: \bar{Z}_{\infty}^{\text {tors }} \rightarrow \overline{\mathscr{L}}$ be the composite of $\theta$ with the natural map $\bar{Z}_{\infty}^{\text {tors }} \rightarrow \mathcal{R}^{\times}$induced by the action of $G$ on $\mathcal{R}$. Denote by $\mathrm{r}_{p}^{\text {xtors }}$ the maximal torsion subgroup of $\mathrm{r}_{p}^{\times}$. For any $\kappa \in \mathcal{X}(\mathcal{I})$, define

$$
\theta_{\kappa}:=\kappa \circ \theta: \mathcal{R} \rightarrow \overline{\mathbb{Q}}_{p} .
$$

By [7], Corollary 2.5, if $\kappa \in \mathcal{A}(\mathcal{I})$ and $\theta$ restricted to $\mathrm{r}_{p}^{\text {xtors }}$ is the character $x \mapsto x^{v(\kappa)}$, then $\theta_{\kappa}(T(\mathfrak{q}))$ are algebraic numbers for all prime ideals $q$ and there exists a $p$-nearly ordinary eigenform

$$
f_{\kappa} \in S_{k(\kappa), w(\kappa)}\left(U_{0}(\mathfrak{n} C(\kappa)), \epsilon_{W} \psi \omega^{-(n(\kappa)+2 v(\kappa))}, \mathbb{C}\right)
$$

(unique up to multiplication by constants) such that $f_{\kappa} \mid T(\mathfrak{q})=\theta_{\kappa}(T(\mathfrak{q})) f_{\kappa}$, where $\omega$ is the Teichmüller character and if $n(\kappa)+2 v(\kappa)=m t$ with $m \in \mathbb{Z}$, then $\omega^{-(n(\kappa)+2 v(\kappa))}:=\omega^{-m}$. Conversely, if $\alpha>0$ and $f \in S_{k, w}\left(U_{1}\left(\mathfrak{n} p^{\alpha}\right), \mathbb{C}\right)$ is a $p$-nearly ordinary eigenform, then there exists $\kappa \in \mathcal{A}(\mathcal{I})$ and $\theta$ as above such that $f$ is a constant multiple of $f_{\kappa}$. 


\section{3. $\Lambda$-adic modular symbols}

3.1. Classical modular symbols. Fix a quaternion algebra $\boldsymbol{B} / F$ which is unramified at all finite places and denote by $r \in\{0, \ldots, d\}$ the number of archimedean places where $\boldsymbol{B}$ is split. Define the modular variety associated to an open compact subgroup $U$ of $\widehat{\boldsymbol{B}}^{\mathrm{x}}$ to be the complex variety

$$
X(U):=U C_{\infty} \backslash \backslash \boldsymbol{G}_{\mathbb{A}} / \boldsymbol{G}_{\mathbb{Q}}
$$

By strong approximation,

$$
X(U) \simeq \coprod_{\lambda=1}^{h(U)} \Gamma^{\lambda}(U) \backslash \mathfrak{S}^{r}
$$

Suppose that $E$ is a (right or left) $\mathcal{O}\left[\Gamma^{\lambda}(U)\right]$-module for all $\lambda$. Denote by $\mathcal{E}$ the coefficient system on $X(U)$ associated to $E$. Then

$$
H^{r}(X(U), \mathcal{E}) \simeq \bigoplus_{\lambda=1}^{h(U)} H^{r}\left(\Gamma^{\lambda}(U) \backslash \mathfrak{S}^{r}, \mathcal{E}\right) \simeq \bigoplus_{\lambda=1}^{h(U)} H^{r}\left(\bar{\Gamma}^{\lambda}(U), E\right)
$$

For any $\omega \in H^{r}(X(U), \mathcal{E})$, write $\omega_{\lambda}$ for its projection to $H^{r}\left(\Gamma^{\lambda}(U) \backslash \mathfrak{S}^{r}, \mathcal{E}\right)$.

Definition 3.1. The group of modular symbols on $X(U)$ associated to $E$ is the group $H_{\mathrm{cpt}}^{r}(X(U), \mathcal{E})$ of cohomology with compact support.

Suppose that $E$ is a right $t_{\lambda} \Delta_{0}\left(\mathfrak{n} p^{\alpha}\right) t_{\lambda}^{-1} \cap \boldsymbol{G}_{\mathbb{Q}}$ for all $\lambda$. Define an action of the Hecke algebra $R\left(S\left(p^{\alpha}\right), \Delta_{0}\left(\mathfrak{n} p^{\alpha}\right)\right)$ by the formula

$$
\omega_{\lambda}(z)\left|T(x):=\sum_{j} \omega_{\lambda}\left(\alpha_{\lambda, j} z\right)\right| \alpha_{\lambda, j} \in H^{r}\left(\Gamma^{\mu}\left(p^{\alpha}\right) \backslash \mathfrak{S}^{r}, \mathcal{E}\right)
$$

(same notation as in $\S 2.2$ ). Equivalently, identifying $\omega_{\lambda}$ with a $d$-cocycle by the above isomorphism, $T(x)$ can be defined as in [2] by the formula

$$
\left(\omega_{\lambda}\right)\left|T(x)\left(\gamma_{0}, \ldots, \gamma_{d}\right):=\sum_{j} \omega_{\lambda}\left(t_{j}\left(\gamma_{0}\right), \ldots, t_{j}\left(\gamma_{d}\right)\right)\right| \alpha_{\lambda, j},
$$

where the mappings $t_{j}: \Gamma^{\mu}\left(p^{\alpha}\right) \rightarrow \Gamma^{\lambda}\left(p^{\alpha}\right)$ are defined for $\gamma \in \Gamma^{\mu}\left(p^{\alpha}\right)$ by the equations $\Gamma_{\lambda} \alpha_{\lambda, j} \gamma=\Gamma_{\lambda} \alpha_{\lambda, l}$ and $\alpha_{\lambda, j} \gamma=t_{j}(\gamma) \alpha_{\lambda, l}$. The group of modular symbols $H_{\text {cpt }}^{r}\left(X\left(S\left(p^{\alpha}\right)\right), \mathcal{E}\right)$ is an $R\left(S\left(p^{\alpha}\right), \Delta_{0}\left(\mathfrak{n} p^{\alpha}\right)\right)$-module if $E$ is.

If $r \geq 1$, the modular symbol $\omega(f)$ associated to $f \in S_{k, w, I_{\boldsymbol{B}}}\left(S\left(p^{\alpha}\right), \boldsymbol{B}, \mathbb{C}\right)$ can be described as follows. For any ring $R$, let $L(n, R)$ be the $R$-module of homogeneous 
polynomials in $2 r$ variables $X=\left(X_{\sigma}\right)_{\sigma \in I}$, and $Y=\left(Y_{\sigma}\right)_{\sigma \in I}$ of degree $n_{\sigma}$ in $X_{\sigma}, Y_{\sigma}$. Recall that $\gamma \mapsto \gamma^{*}$ denotes the main involution of $\mathrm{M}_{2}(R)$ and define a right action of $\mathrm{GL}_{2}(F)^{r}$ on $L(n, \mathbb{C})$ by

$$
(P \mid \gamma)(X, Y):=\operatorname{det}(\gamma)^{v} P\left((X, Y) \gamma^{*}\right)
$$

where $(X, Y) \gamma^{*}$ is matrix multiplication and the usual multi-index notation is used. Denote by $L(n, v, \mathbb{C})$ the right representation of $\boldsymbol{B}$ obtained composing the above action with the injection $\boldsymbol{B}^{\times} \hookrightarrow \mathrm{GL}_{2}(F)^{r}$. The differential form

$$
\omega\left(f_{\lambda}\right)(z):=f(z)(z X+Y)^{k-2} d z
$$

(usual multi-index notation) satisfies the transformation formula for any $\gamma \in \Gamma^{\lambda}\left(p^{\alpha}\right)$ :

$$
\left(\omega\left(f_{\lambda}\right)\right)(\gamma(z)) \mid \gamma=\omega\left(f_{\lambda}\right)(z) .
$$

Hence, by [11], $\omega\left(f_{\lambda}\right) \in H^{r}\left(\Gamma^{\lambda}\left(p^{\alpha}\right) \backslash \mathfrak{S}^{d}, \mathscr{L}(n, v, \mathbb{C})\right)$, where $\mathscr{L}(n, v, \mathbb{C})$ is the coefficient system on $\Gamma^{\lambda}\left(p^{\alpha}\right) \backslash \mathfrak{H}^{r}$ associated to $L(n, v, \mathbb{C})$. If $\boldsymbol{B}=M_{2}(F)$, since $f_{\lambda}$ is a cusp form, it can be proved that $\omega\left(f_{\lambda}\right)$ has compact support. Hence, we always have

$$
\omega(f):=\left(\omega\left(f_{1}\right), \ldots, \omega\left(f_{h}\right)\right) \in H_{\mathrm{cpt}}^{r}\left(X\left(S\left(p^{\alpha}\right), \mathscr{L}(n, v, \mathbb{C})\right) .\right.
$$

For any character $\epsilon$ as above, write $L(n, v, \epsilon, R)$ for the $\Delta_{0}(\mathfrak{n})$-module $L(n, v, R)$ with the action of $\Delta_{0}(\mathfrak{n})$ twisted by $\epsilon$, that is, denoting by $\left.\right|_{\epsilon}$ this new action: $\left.P\right|_{\epsilon} \gamma:=\epsilon(\gamma) P \mid \gamma$ for $\gamma \in \Delta_{0}(\mathfrak{m})$. If $f_{\lambda} \in S_{k, w}\left(S_{0}\left(p^{\alpha}\right), \epsilon, \mathbb{C}\right)$, then $\omega\left(f_{\lambda}\right) \in$ $H^{r}\left(\Gamma_{0}^{\lambda}\left(p^{\alpha}\right), \mathscr{L}(n, v, \epsilon, \mathbb{C})\right)$, where $\mathscr{L}(n, v, \epsilon, \mathbb{C})$ is the coefficient system associated to $L(n, v, \epsilon, \mathbb{C})$. Hence

$$
\omega(f) \in H_{\text {cpt }}^{r}\left(X\left(S_{0}\left(p^{\alpha}\right)\right), \mathscr{L}(n, v, \epsilon, \mathbb{C})\right) .
$$

A straightforward calculation shows that the map $f \mapsto \omega(f)$ is equivariant for the action of $R\left(S\left(p^{\alpha}\right), \Delta_{0}\left(\mathfrak{n} p^{\alpha}\right)\right)$.

3.2. $\boldsymbol{\Lambda}$-adic modular symbols. We assume in this section that $r \leq 1$, so that $\boldsymbol{B}$ is a division algebra. Recall also that $\boldsymbol{B}$ is assumed to be unramified at all finite places of $F$.

Define $e=e_{S}:=S \cap F^{\times}$. Then $\bar{Z}_{\alpha}=\left(\mathrm{r}_{p} / p^{\alpha} \mathrm{r}_{p}\right)^{\times} / e$, so $G=\mathrm{r}_{p}^{\times} \times \mathrm{r}_{p}^{\times} / \bar{e}$, where $\bar{e}$ is the closure of $e$ in $\mathrm{r}_{p}^{\times}$. It follows that $G \simeq\left(\mathrm{r}_{p}^{\times} \times \mathrm{r}_{p}^{\times}\right) / \bar{e}$ via the map $(x, y) \mapsto(x y, y)$. Embed diagonally $\bar{e}$ in $\mathrm{GL}_{2}\left(\mathrm{r}_{p}\right)$ and call $C$ the image. Let $N$ be the standard lower unipotent subgroup of $\mathrm{GL}_{2}\left(\mathrm{r}_{p}\right)$. Define the sets

$$
X_{\alpha}:=S\left(p^{\alpha}\right) \mathrm{r}^{\times} \backslash S \mathrm{r}^{\times} \quad \text { and } \quad X:=\lim _{\longleftarrow} X_{\alpha} .
$$

To describe these sets, let $N_{\alpha}=\left(\begin{array}{ll}a & b \\ c & d\end{array}\right) \in \mathrm{GL}_{2}\left(\mathrm{r}_{p}\right)$ be such that $a, d=1 \bmod p^{\alpha}$ and $c=0 \bmod p^{\alpha}$. We have a surjective map $S\left(p^{\alpha}\right) \backslash S \rightarrow X_{\alpha}$. Note that $S\left(p^{\alpha}\right) \backslash S=$ 
$N_{\alpha} \backslash \mathrm{GL}_{2}\left(\mathrm{r}_{p}\right)$, and that the kernel of the above map is $N_{\alpha} \backslash N_{\alpha} r^{\times}$, so we have a bijection of sets $N_{\alpha} \mathrm{r}^{\times} \backslash \mathrm{GL}_{2}\left(\mathrm{r}_{p}\right) \rightarrow X_{\alpha}$. Finally, since $N C$ is the closure of $\cap N_{\alpha} \mathrm{r}^{\times}$in $\mathfrak{r}_{p}$, we see that $X$ is canonically identified with $N C \backslash \mathrm{GL}_{2}\left(\mathrm{r}_{p}\right)$.

Let $F_{p}=F \otimes_{\mathbb{Q}} \mathbb{Q}_{p}=\prod_{\mathfrak{p} \mid p} F_{\mathfrak{p}}$ and $Y:=N\left(F_{p}\right) C \backslash \mathrm{GL}_{2}\left(F_{p}\right)$, where $N\left(F_{p}\right)$ is the group of lower triangular matrices with entries in $F_{p}$ and diagonal entries 1. Write $\mathfrak{r}_{p}=\prod_{\mathfrak{p} \mid p} \mathfrak{r}_{\mathfrak{p}}$, where $\mathfrak{r}_{\mathfrak{p}}$ is the completion of $\mathfrak{r}$ at $\mathfrak{p}$. Define $\left(\mathrm{r}_{\mathfrak{p}}^{2}\right)^{\prime}$ to be the set of primitive vectors of $\mathrm{r}_{\mathfrak{p}}^{2}$, that is, the pairs of elements $(a, b) \in \mathrm{r}_{p}^{2}$ such that at least one of $a$ and $b$ does not belong to $\mathfrak{p}$. Set $\left(\mathrm{r}_{p}^{2}\right)^{\prime}:=\prod_{\mathfrak{p} \mid p}\left(\mathrm{r}_{\mathfrak{p}}^{2}\right)^{\prime}$. The map

$$
g=\left(\begin{array}{ll}
a & b \\
c & d
\end{array}\right) \longmapsto((a, b), \operatorname{det}(g))
$$

defines a bijection between $X$ and $\bar{e} \backslash\left(\left(\mathrm{r}_{p}^{2}\right)^{\prime} \times \mathrm{r}_{p}^{\times}\right)$, where the action of $e \in \overline{\mathrm{e}}$ is $e \cdot((x, y), z)=\left((e x, e y), e^{2} z\right)$.

Define $\pi_{\mathfrak{p}}$ to be the element in $\mathrm{GL}_{2}(\widehat{F})$ whose $\mathfrak{p}$-component is $\left(\begin{array}{ll}1 & 0 \\ 0 & p\end{array}\right)$ and is 1 outside $\mathfrak{p}$. Note that $\pi_{\mathfrak{p}}$ normalizes $N\left(F_{p}\right)=\prod_{\mathfrak{p}^{\prime} \mid p} N\left(F_{\mathfrak{p}^{\prime}}\right)$ because $\left(\begin{array}{ll}1 & 0 \\ 0 & \mathfrak{p}\end{array}\right)$ normalizes $N\left(F_{\mathfrak{p}}\right)$. Hence, it is possible to define an action of $\pi_{\mathfrak{p}}$ on $Y$ by letting $\pi_{\mathfrak{p}}$ act on its $\mathfrak{p}$-component as

$$
N\left(F_{\mathfrak{p}}\right) g * \pi_{\mathfrak{p}}=N\left(F_{\mathfrak{p}}\right) \pi_{\mathfrak{p}}^{-1} g \pi_{\mathfrak{p}} .
$$

Identify $Y$ with $\bar{e} \backslash\left(\left(F_{p}^{2}\right)^{\prime} \times F_{p}^{\times}\right)$, where $\left(F_{p}^{2}\right)^{\prime}:=\left\{(x, y) \in F_{p}^{2}: x y \neq 0\right\}$ via the $\operatorname{map} \gamma=\left(\begin{array}{ll}a & b \\ c & d\end{array}\right) \mapsto((a, b), \operatorname{det}(\gamma))$. Then

$$
((x, y), z) * \pi_{\mathfrak{p}}=((x, \mathfrak{p} y), z),
$$

where for any $y=\prod_{\mathfrak{p}^{\prime} \mid p} y_{\mathfrak{p}}$, write $\mathfrak{p} y:=\prod_{\mathfrak{p}^{\prime} \mid p, \mathfrak{p}^{\prime} \neq \mathfrak{p}} y_{\mathfrak{p}^{\prime}} \times \mathfrak{p} y_{\mathfrak{p}}$. In particular, $\pi_{\mathfrak{p}}$ does not affect the determinant of the matrix.

Let $G^{\pi}$ be the semigroup generated by $\mathrm{GL}_{2}\left(\mathrm{r}_{p}\right)$ and $\pi_{\mathfrak{p}}$ for all divisors $\mathfrak{p}$ of $p$. Using that any element $s \in G^{\pi}$ can be expressed as a word in terms of $\mathrm{GL}_{2}\left(\mathrm{r}_{p}\right)$ and $\pi_{\mathfrak{p}}$, and that the actions of the $\pi_{\mathfrak{p}}$ 's commute, extend the $*$ action to $G^{\pi}$ by letting any $\pi_{\mathfrak{p}}$ act through $*$ and elements of $\mathrm{GL}_{2}\left(\mathfrak{r}_{p}\right)$ through right multiplication, so that

$$
N\left(F_{p}\right) g * s=N\left(F_{p}\right) \prod_{\mathfrak{p} \mid p} \pi_{\mathfrak{p}}^{-c(\mathfrak{p})} g s
$$

for any $N\left(F_{p}\right) g \in Y$ and $s \in G^{\pi}$, where $c(\mathfrak{p})$ is the number of times $\pi_{\mathfrak{p}}$ appears in the expression of $s$. Since this number does not depend on the specific expression we chose, the action of $G^{\pi}$ on $Y$ is well defined.

Let $Y^{\prime}$ denote the smallest subset of $Y$ containing $X$ and being stable under $G^{\pi}$. Define $\mathbb{D}_{X}$ (respectively, $\mathbb{D}_{Y^{\prime}}$ ) to be the $\mathcal{O}$-module of $\mathcal{O}$-valued measures on $X$ (respectively, on $Y^{\prime}$ ). For $\gamma \in G^{\pi}$ and $\mu \in \mathbb{D}_{Y^{\prime}}$, define $\mu * \gamma$ by the integration formula

$$
\int_{Y^{\prime}} \varphi(\eta) d(\mu * \gamma)(\eta):=\int_{Y^{\prime}} \varphi(\eta * \gamma) d \mu(\eta),
$$


where $\varphi$ is any $\mathbb{C}_{p}$-valued step function on $Y^{\prime}$. Denote by $\mathbb{D}_{X} \stackrel{i}{\rightarrow} \mathbb{D}_{Y^{\prime}}$ the canonical inclusion defined by extending measures by zero and by $\mathbb{D}_{Y^{\prime}} \stackrel{p}{\rightarrow} \mathbb{D}_{X}$ the canonical projection map. If $\mu \in \mathbb{D}$ and $s \in G^{\pi}$, define

$$
\mu * s=p(i(\mu) * s) .
$$

Since by [2], Lemma 3.1, the kernel of $p$ is stable under $G^{\pi}$, the action is well defined.

By the choice of $t_{\lambda}$ in $\S 2, \Gamma^{\lambda}\left(p^{\alpha}\right) \subseteq \mathrm{GL}_{2}\left(\mathrm{r}_{p}\right)$ for all $\lambda$. Hence, $\mathbb{D}_{X}$ is a right $\mathcal{O}\left[\Gamma^{\lambda}\left(p^{\alpha}\right)\right]$-module for all $\lambda$. Denote by $\mathscr{D}_{X}$ the coefficient system associated to $\mathbb{D}_{X}$ and set

$$
\mathbb{W}:=H^{r}\left(X(S), D_{X}\right)
$$

Remark 3.2. Since $\mathrm{SO}_{2}(\mathbb{R})$ is compact and isomorphic to the unit circle $\mathbb{C}^{1}$ and the $\Gamma^{\lambda}$ are discrete, the stabilizer $\left(\bar{\Gamma}^{\lambda}\right)_{z_{0}}$ of any element $z_{0} \in \mathfrak{S}^{r}$ is a finite cyclic group. Since the groups $\bar{\Gamma}^{\lambda}$ are torsion-free, it follows that if $\gamma \in \Gamma^{\lambda}$ stabilizes an element $z_{0} \in \mathfrak{S}^{r}$, then $\gamma$ belongs to the center of $\Gamma^{\lambda}$ and hence acts trivially on $\mathbb{D}_{X}$. It follows that the sheaf $\mathscr{D}_{X}$ is well defined.

Lemma 3.3. Let $Z$ be a topological space that is an inverse limit of finite discrete topological spaces $Z_{\alpha}$ for $\alpha$ in some indexing set. Then the space of $\mathcal{O}$-valued measures on $Z$ is isomorphic to $\lim \operatorname{Fns}\left(Z_{\alpha}, \mathcal{O}\right)$ where $\operatorname{Fns}\left(Z_{\alpha}, \mathcal{O}\right)=\mathcal{O}^{Z_{\alpha}}$ is the space of continuous $\mathcal{O}$-valued functions on $Z_{\alpha}$.

Proof. If $\phi \in \lim \operatorname{Fns}\left(Z_{\alpha}, \mathcal{O}\right)$, then $\mu$ can be written as a compatible sequence of the form $\left\{\sum_{x \in Z_{\alpha}} a_{x} \cdot x\right\}_{\alpha}$. Let $p_{\alpha}: Z \rightarrow Z_{\alpha}$ be the natural projection map and for each $x \in Z_{\alpha}$ set $U_{\alpha, x}=p_{\alpha}^{-1}(x)$. Then this is isomorphic to the space of $\mathcal{O}$-valued measures on $Z$ by the map

$$
\phi \longmapsto \mu \quad \text { such that } \mu\left(U_{\alpha, x}\right)=a_{g} .
$$

This defines a measure due to the compatibility of the sequence.

For each $\alpha$, let $p_{\alpha}: X\left(S\left(p^{\alpha}\right)\right) \rightarrow X(S)$. Let $\mathcal{F}_{\alpha}=p_{\alpha *} \mathcal{O}$ be the direct image of the constant sheaf $\mathcal{O}$ on $X(S)$. Fix a point $x \in X(S)$ and define $Y_{\alpha}=Y_{\alpha, x}$ to be the fiber $p_{\alpha}^{-1}(x)$ of $x$ under $p_{\alpha}$. By Lemma 3.3, $\lim ^{\mathcal{O}^{Y_{\alpha}}}$ is the space of $\mathcal{O}$-valued measures on the space $\lim _{\longleftarrow} Y_{\alpha}$. Now, for the double coset $\mathrm{GL}_{2}(F) x S S_{\infty}$ in $X(S)$, there is a natural map

$$
S\left(p^{\alpha}\right) \backslash S=S\left(p^{\alpha}\right)_{p} \backslash \mathrm{GL}_{2}\left(\mathrm{r}_{p}\right) \longrightarrow Y_{\alpha}
$$

given by

$$
S\left(p^{\alpha}\right)_{p} z \longmapsto S\left(p^{\alpha}\right) S_{\infty} x z \mathrm{GL}_{2}(F)
$$


This map induces a bijection from $S\left(p^{\alpha}\right)\left(\mathrm{r}^{\times} \cap S S_{\infty}\right) \backslash \mathrm{GL}_{2}\left(\mathrm{r}_{p}\right)$ to $Y_{\alpha}$. Hence the inverse limit $\lim _{\longleftarrow} Y_{\alpha}$ can be identified with $N C \backslash \mathrm{GL}_{2}\left(\mathrm{r}_{p}\right)$ which is finally identified with $X$.

Lemma 3.4. The sheaves $\mathscr{D}_{X}$ and $\underset{\alpha}{\lim } \mathcal{F}_{\alpha}$ on $X(S)$ are isomorphic.

Proof. Let $U \in X(S)$ be an open set. For each $\alpha$ and $w \in Y_{\alpha}$, let $X_{\alpha, w} \subset X$ be the inverse image of $w$ under the natural projection map $X \rightarrow Y_{\alpha}$. Let $u \in U$, then choose $x \in \mathrm{GL}_{2}\left(F_{\mathbb{A}}\right)$ such that $u=S S_{\infty} x \mathrm{GL}_{2}(F)$. If $s \in \mathscr{D}_{X}(U)$ is a section in $\mathscr{D}_{X}$, we can express $s(u)=S S_{\infty}(x, \mu(x)) \mathrm{GL}_{2}(F)$ for some $\mu \in \mathbb{D}_{X}$, depending on $s$. In fact, since $s$ is a locally constant section, this expression is valid in a neighborhood $U_{u}$ of $u$. Then define a map:

$$
\begin{array}{cll}
D_{X}(U) & \stackrel{\phi_{\alpha}(U)}{\longmapsto} & \mathcal{F}_{\alpha}(U)=\mathcal{O}\left(p_{\alpha}^{-1}(U)\right) \\
s & \longmapsto & u \mapsto \sum_{w \in Y_{\alpha}} \mu(x)\left(X_{\alpha, w}\right) .
\end{array}
$$

This map is independent of the choice of $x$ since a different representative $x^{\prime}$ of the double coset $u$ would yield the same measure $\mu(x)$ because $s$ is a section on $X(S)$.

The compatible maps $\phi_{\alpha}$ then give rise to a map

$$
\phi(U): \mathscr{D}_{X}(U) \longrightarrow\left(\lim _{\alpha} \mathcal{F}_{\alpha}\right)(U)
$$

At the level of the stalks this map is the isomorphism in (4). It follows that $\phi$ is an isomorphism of sheaves.

Proposition 3.5. We have a canonical isomorphism

$$
\mathbb{W} \cong \lim _{\longleftarrow} H^{r}\left(X\left(S\left(p^{\alpha}\right)\right), \mathcal{O}\right) .
$$

Proof. If $r=0$ the result is obvious so we can assume that $r=1$. We know from Lemma 3.4 and Theorem 4.5 in [5] that there is a short exact sequence

$$
0 \longrightarrow \lim ^{(1)}\left\{H^{0}\left(X\left(S\left(p^{\alpha}\right)\right), \mathcal{O}\right)\right\} \longrightarrow \mathbb{W} \longrightarrow \lim _{\longleftarrow} H^{1}\left(X\left(S\left(p^{\alpha}\right)\right), \mathcal{O}\right) \longrightarrow 0 .
$$

So we need to show that the kernel is zero. Let $H_{\alpha}=H^{0}\left(X\left(S\left(p^{\alpha}\right)\right), \mathcal{O}\right)$ and we know that $H_{\alpha} \equiv \bigoplus H^{0}\left(\bar{\Gamma}^{\lambda}\left(p^{\alpha}\right), \mathcal{O}\right)$. The action of $\bar{\Gamma}^{\lambda}\left(p^{\alpha}\right)$ on $\mathcal{O}$ is trivial, so the zeroth cohomology group is just $\mathcal{O}$. Hence if $\alpha \geq \beta$, the transition map $p_{\alpha \beta}: H_{\alpha} \rightarrow H_{\beta}$ in the inverse system $\left\{H_{\alpha}\right\}$ is just the inflation map and is given by multiplication by $\left[\bar{\Gamma}^{\lambda}\left(p^{\beta}\right): \bar{\Gamma}^{\lambda}\left(p^{\alpha}\right)\right]$ in the $\lambda$-th component. If we fix $\beta$ then the filtration given by images of $H_{\alpha}$ in $H_{\beta}$, for $\alpha \geq \beta$, stabilizes if $p$ does not divide the indices above and is a sub-filtration of $\mathcal{O} \supset \pi \mathcal{O} \supset \pi^{2} \mathcal{O} \cdots$ otherwise. In the first case $\left\{H_{\alpha}\right\}$ satisfies the Mittag-Leffler condition and in the second case $\mathcal{O}$ is complete under the filtration. Hence from [5], Corollary 4.3, in the first case and [5], Proposition 4.2, in the second case, the first derived functor of the inverse limit is 0 in either case. 
Since $t_{\lambda} \Delta_{0}(\mathfrak{n}) t_{\lambda}^{-1} \cap \boldsymbol{G}_{\mathbb{Q}} \subseteq G^{\pi}$ for all $\lambda=1, \ldots, h$, it follows that $\mathbb{W}$ is an $R_{\mathcal{O}}\left(S, \Delta_{0}(\mathfrak{n})\right):=\mathcal{O} \otimes_{\mathbb{Z}} R\left(S, \Delta_{0}(\mathfrak{n})\right)$-module. Let $G$ act on $X$ by left multiplication. Define $G^{\prime}$ to be the multiplicative subset of $(\mathrm{r} \times \mathrm{r}) / \mathrm{r}^{\times}$consisting of pairs of elements $(x, y)$ such that $x$ and $y$ are prime to $p$. The map $(a, d) \mapsto \omega\left(a_{p}^{n+2 v}\right)^{-1} T\left(\begin{array}{ll}a & 0 \\ 0 & d\end{array}\right)$ for $(a, d) \in G^{\prime}$ considered in $\S 2.2$ is multiplicative, hence extends to an $\mathcal{O}$-algebra homomorphism $\mathcal{O}\left[G^{\prime}\right] \rightarrow R_{\mathcal{O}}\left(S, \Delta_{0}(\mathfrak{n})\right)$. On the other hand, $G^{\prime} \subseteq G$, hence $\mathcal{O}\left[G^{\prime}\right]$ embeds naturally in $\tilde{\Lambda}=\mathcal{O} \llbracket G \rrbracket$. Form the $\widetilde{\Lambda}$-algebra

$$
\mathscr{H}:=R_{\mathcal{O}}\left(S, \Delta_{0}(\mathfrak{m})\right) \otimes_{\mathcal{O}\left[G^{\prime}\right]} \tilde{\Lambda} .
$$

Since the action of $G^{\prime}$ on $\mathbb{W}$ extends to a continuous action of $G$, it follows that $\mathbb{W}$ is an $\mathscr{H}$-module.

From the fact that $h_{2 t, t}\left(S\left(p^{\alpha}\right), \mathcal{O}\right)$ is generated over $\mathcal{O}$ by $T(\mathfrak{q})$ for all prime ideals $q$ and those operators coming from the action of $G_{\alpha}$, it follows that there are surjective homomorphisms of $\widetilde{\Lambda}$-algebras $\mathscr{H} \rightarrow h_{2 t, t}\left(S\left(p^{\infty}\right), \mathcal{O}\right)$ and $\mathscr{H} \rightarrow \mathcal{R}$.

Define a subset $X^{\prime}$ of $X$ as follows:

$$
X^{\prime}=\left\{x=N C\left(\begin{array}{ll}
a & b \\
c & d
\end{array}\right) \in X \mid a \in \mathrm{r}_{p}^{\times}\right\} .
$$

It is easy to check that the definition does not depend on the choice of the representative matrix used to define it and that $X^{\prime}$ can be identified with the set $\bar{e} \backslash\left(\mathrm{r}_{p}^{\times} \times \mathrm{r}_{p} \times \mathrm{r}_{p}^{\times}\right)$ under the above identification between $X$ and $\bar{e} \backslash\left(\left(\mathrm{r}_{p}^{2}\right)^{\prime} \times \mathrm{r}_{p}^{\times}\right)$. From now on denote elements of $X$ by $((x, y), z)$, where $(x, y)$ is the first row of the matrix and $z$ is its determinant.

Let $P_{n, v, \epsilon}$ be an arithmetic point of weight $(n, v)$ and character $\epsilon$ factoring through $Z_{\alpha}$. Define the specialization map $\rho_{n, v, \epsilon}: \mathbb{D}_{X} \rightarrow L(n, v, \epsilon, \mathcal{O})$ at $P$ by

$$
\mu \longmapsto \rho_{n, v, \epsilon}(\mu):=\int_{X^{\prime}} z^{v} \epsilon(x)(x Y-y X)^{n} d \mu(x, y, z) .
$$

Suppose that the conductor of $\epsilon$ is $p^{\alpha}$ for some non-negative integer $\alpha$. A simple computation shows that

$$
\rho_{n, v, \epsilon}(\mu * \gamma)=\left.\rho_{n, v, \epsilon}(\mu)\right|_{\epsilon} \gamma
$$

for $\gamma \in \mathrm{GL}_{2}\left(\mathrm{r}_{p}\right) \cap \Delta_{0}\left(p^{\alpha}\right)$. It follows that the specialization map $\rho_{n, v, \epsilon}$ is $\mathrm{GL}_{2}\left(\mathrm{r}_{p}\right) \cap$ $\Delta_{0}\left(p^{\alpha}\right)$-equivariant. Letting $K:=\operatorname{Frac}(\mathcal{O})$, there $\operatorname{are~} \mathrm{GL}_{2}\left(\mathrm{r}_{p}\right) \cap \Delta_{0}\left(p^{\alpha}\right)$-equivariant maps

$$
\rho_{n, v, \epsilon}: \mathbb{W} \longrightarrow \mathbb{W}_{n, v, \epsilon}:=H^{r}\left(X\left(S\left(p^{\alpha}\right)\right), \mathscr{L}(n, v, \epsilon, \mathcal{O})\right) .
$$

Proposition 3.6. Let $\Phi \in \mathbb{W}$.

(1) For any prime ideal $\mathfrak{q}$ of $\mathfrak{r}$ prime to $p$ we have

$$
\rho_{n, v, \epsilon}(\Phi * T(q))=\left(\rho_{n, v, \epsilon}(\Phi)\right) \mid T(q) .
$$

(2) $\rho_{n, v, \epsilon}(\Phi * T(p))=\left(\rho_{n, v, \epsilon}(\Phi)\right) \mid T_{0}(p)$. 
Proof. The equivariance for the action of Hecke operators $T(q)$ in the first statement is immediate because of the $\mathrm{GL}_{2}\left(\mathrm{r}_{p}\right) \cap \Delta_{0}\left(p^{\alpha}\right)$-equivariance of $\rho_{n, v, \epsilon}$. It remains to check the action of $T(p)$. To see this, write $\Gamma^{\lambda} \pi \Gamma^{\lambda}=\bigsqcup_{t} \Gamma^{\lambda} \alpha_{\lambda, t}$ and note that

$$
\begin{aligned}
\rho_{n, v, \epsilon}\left(\Phi * T_{0}(p)\right) & =\int_{X^{\prime}} \sum_{t} z^{v} \epsilon(x)(x Y-y X)^{n} d\left(\Phi * \alpha_{\lambda, t}\right) \\
& =\int_{X^{\prime}} \sum_{t} z^{v} \epsilon(x)(x Y-y X)^{n} d\left(\pi^{-1} \Phi \alpha_{\lambda, t}\right) \\
& =\sum_{t} \int_{X^{\prime}}\left\{p^{v}\right\}^{-1} z^{v} \epsilon(x)(x Y-y X)^{n} \mid \alpha_{\lambda, t} d(\Phi) \\
& =\rho_{n, v, \epsilon}(\Phi) \mid T_{0}(p) .
\end{aligned}
$$

This proves the second formula.

Note that

$$
\mathbb{W}=\prod_{\lambda=1}^{h} \mathbb{W}^{\lambda}, \quad \text { where } \mathbb{W}^{\lambda}=H^{r}\left(\bar{\Gamma}^{\lambda} \backslash \mathfrak{S}^{r}, \mathcal{D}_{X}\right)
$$

and

$$
\mathbb{W}_{n, v, \epsilon}=\prod_{\lambda=1}^{h} \mathbb{W}_{n, v, \epsilon}^{\lambda}, \quad \text { where } \mathbb{W}_{n, v, \epsilon}^{\lambda}:=H^{r}\left(\bar{\Gamma}_{0}^{\lambda}\left(p^{\alpha}\right) \backslash \mathfrak{S}^{r}, \mathscr{L}(n, v, \epsilon, K)\right)
$$

Any element $\Phi \in \mathbb{W}$ will be written as $\left(\Phi_{\lambda}\right)_{\lambda=1, \ldots, h}$ while any element of $\omega \in \mathbb{W}_{n, v, \epsilon}$ will be denoted as $\left(\omega_{\lambda}\right)_{\lambda=1, \ldots, h}$. Define

$$
\Phi_{\lambda, \theta_{n, v, \epsilon}}:=\rho_{n, v, \epsilon}\left(\Phi_{\lambda}\right) \in \mathbb{W}_{n, v, \epsilon}^{\lambda} .
$$

3.3. The Control Theorem. In this section we will state and prove a control theorem for $\mathbb{W}$. We will assume throughout this section that $r \leq 1$, so that $\boldsymbol{B}$ is a division algebra; also recall that $\boldsymbol{B}$ is assumed to be unramified at all finite places.

Fix $\theta: \mathcal{R} \rightarrow \overline{\mathscr{L}}$ (where $\mathscr{L}=\operatorname{Frac}(\Lambda)$ ) and denote as in $\S 2.3$ by $\mathcal{I}$ the integral closure of $\Lambda$ in $\operatorname{Im}(\theta)$. Recall the specialization map

$$
\theta_{\kappa}:=\kappa \circ \theta: \mathcal{R} \longrightarrow \overline{\mathbb{Q}}_{p}
$$

which corresponds to an eigenform $f_{\kappa}$. Let $P_{\kappa}$ be the kernel of $\theta_{\kappa}$. Set

$$
\mathbb{W}_{\kappa}:=\prod_{\lambda=1}^{h} \mathbb{W}_{\kappa}^{\lambda} \quad \text { with } \mathbb{W}_{\kappa}^{\lambda}:=H^{r}\left(\bar{\Gamma}_{0}^{\lambda}\left(p^{\alpha}\right) \backslash \mathfrak{H}^{r}, \mathscr{L}\left(n, v, \epsilon, K_{\kappa}\right)\right),
$$


where

$$
K_{\kappa}:=\text { fraction field of the image of } \theta_{\kappa} .
$$

The map $\theta_{\kappa}$ extends to the localization $\mathcal{R}_{P_{\kappa}}$ of $\mathcal{R}$ at $P_{\kappa}$ and one can intertwine $\theta_{\kappa}$ with the map $\rho_{\kappa}$ defining

$$
\rho_{\kappa}: \mathbb{W} \times \mathcal{R}_{P_{\kappa}} \longrightarrow \mathbb{W}_{\kappa}
$$

by

$$
\sum_{\lambda} \Phi_{\lambda} \times r_{\lambda} \longmapsto \sum_{\lambda} \rho_{\kappa}\left(\Phi_{\lambda}\right) \cdot \theta_{\kappa}\left(r_{\lambda}\right) .
$$

This is well defined because, if $g$ belongs to the free part $W$ of $G$ and is represented by the matrix $\left(\begin{array}{ll}a & 0 \\ 0 & d\end{array}\right) \in \mathrm{GL}_{2}\left(\mathrm{r}_{p}\right)$, then we have for $\mu \in \mathbb{D}_{X}$ that

$$
\rho_{\kappa}(g \mu)=\rho_{\kappa}(g) \rho_{\kappa}(\mu) .
$$

Since $\rho_{\kappa}(\phi g, r)=\rho_{\kappa}(\phi, g r)$ for $g \in W$ and by continuity the same is true for any element in $\Lambda$, the map (7) induces a homomorphism

$$
\rho_{\kappa}: \mathbb{W} \otimes_{\Lambda} \mathcal{R}_{P_{\kappa}} \longrightarrow \mathbb{W}_{\kappa}
$$

which is Hecke-equivariant.

For any $\mathscr{H}$-module $M$, let $M^{\text {ord }}$ denote its ordinary part, that is, the maximal subspace of $M$ on which the $T(p)$ operator acts as a unit. Let $h: \mathscr{H} \rightarrow \mathcal{R}$ be the natural map obtained by the action of Hecke operators on $\Lambda$-adic cusp forms. For any arithmetic point $\kappa$, let $h_{\kappa}$ be the composition of $h$ with the localization morphism $\mathcal{R} \rightarrow \mathcal{R}_{P_{\kappa}}$. For any $\mathscr{H} \otimes_{\Lambda} \mathcal{R}_{P_{\kappa}}$-module $M$, let

$$
M^{h_{\kappa}}=\left\{m \in M \mid(T(\mathfrak{q}) \otimes 1) m=\left(1 \otimes h_{\kappa}(T(\mathfrak{q}))\right) \cdot m \text { for all prime ideals } \mathfrak{q} \text { in } \mathfrak{r}\right\}
$$

denote the $h_{\kappa}$-eigenspace of $M$. If $f_{\kappa}$ is a classical eigenform for an arithmetic point $\kappa$, let

$$
\mathbb{W}_{\kappa}^{f_{\kappa}}=\left\{\phi \in \mathbb{W}_{\kappa} \mid T_{0}(\mathfrak{q}) \phi=a_{\mathfrak{q}}(g) \phi\right\}
$$

denote the $f_{\kappa}$-eigenspace of $\mathbb{W}_{\kappa}$, where $a_{\mathfrak{q}}(g)$ is the eigenvalue of the $T_{0}(\mathfrak{q})$ operator on $f_{\kappa}$. Hence, there is a map

$$
\rho_{\kappa}:\left(\mathbb{W} \otimes_{\Lambda} \mathcal{R}_{P_{\kappa}}\right)^{h_{\kappa}} \longrightarrow \mathbb{W}_{\kappa}^{f_{\kappa}}
$$

In the case $r=1$ we also have an action of complex conjugation $\tau$ on $X\left(S\left(p^{\alpha}\right)\right)$. The action of $\tau$ corresponds to the action on $X\left(S\left(p^{\alpha}\right)\right)$ deduced from the function $z \mapsto$ $-\bar{z}$ on the complex upper half-plane $\mathfrak{S}$ by the canonical projection $\mathfrak{H} \rightarrow X\left(S\left(p^{\alpha}\right)\right)$ (here $\bar{z}$ is the complex conjugate of $z$ ). For any module $M$ over which $\tau$ acts and for each sgn $\in\{ \pm 1\}$, let $M^{\text {sgn }}$ denote the sgn-eigenmodule of $M$ for the action of $\tau$.

We are now ready to state our main theorem. We need to make the assumption that the generators of $P_{\kappa}$ are regular in $\mathcal{R}_{P_{\kappa}}$ or equivalently, since $\mathcal{R}_{P_{\kappa}}$ is a local ring, that 
$\mathcal{R}_{P_{\kappa}}$ is regular. This condition is a generalization of the notion of primitive eigenforms to the nearly ordinary setting. See Remark 3.8 for a more precise discussion of this issue.

Theorem 3.7 (Control Theorem). Let $\kappa \in \mathcal{A}(\mathcal{I})$ be an arithmetic point of weight $\left(n_{\kappa}, v_{\kappa}\right)$ and character $\epsilon_{\kappa}$. Assume that $\mathcal{R}_{P_{\kappa}}$ is a regular local ring. Then the map

$$
\rho_{\kappa}:\left(\mathbb{W} \otimes_{\Lambda} \mathcal{R}_{P_{\kappa}}\right)^{h_{\kappa}} / P_{\kappa}\left(\mathbb{W} \otimes_{\Lambda} \mathcal{R}_{P_{\kappa}}\right)^{h_{\kappa}} \longrightarrow \mathbb{W}_{\kappa}^{f_{\kappa}}
$$

is an isomorphism. If $r=1$, then for each $\operatorname{sgn} \in\{ \pm 1\}$ we also have an isomorphism

$$
\rho_{\kappa}:\left(\mathbb{W} \otimes_{\Lambda} \mathcal{R}_{P_{\kappa}}\right)^{h_{\kappa}, \mathrm{sgn}} / P_{\kappa}\left(\mathbb{W} \otimes_{\Lambda} \mathcal{R}_{P_{\kappa}}\right)^{h_{\kappa}, \mathrm{sgn}} \longrightarrow \mathbb{W}_{\kappa}^{f_{\kappa}, \mathrm{sgn}} .
$$

Remark 3.8. As mentioned before Theorem 3.7, the condition that $\mathcal{R}_{P_{\kappa}}$ is a regular local ring is a suitable generalization of the concept of primitive eigenforms in the nearly ordinary setting. To be more precise, note that, since $P_{n, v}$ is generated by a regular sequence in $\Lambda$, then $P_{\kappa}$ is generated by a regular sequence in $\mathcal{R}$ if the extension of local rings $\mathcal{R}_{P_{k}} / \Lambda_{P_{n, v, \epsilon}}$ is unramified. In the case $F=\mathbb{Q}$, the assumption that $f$ is a primitive form implies that the corresponding prime $P_{\kappa}$ is unramified over $P_{n, 0, \epsilon}$ (recall that in the rational case one always has $v=0$ ). Similar conditions are also true in the case of families of Hilbert modular forms of parallel weight thanks to [13], §12.7.7. As an alternative choice of $P_{\kappa}$, one could consider the reduced Hecke algebra $\mathcal{R}^{\text {red }}$ generated by operators $T(\mathfrak{q})$ and $T(\mathfrak{q}, \mathfrak{q})$ for primes $\mathfrak{q} \nmid p \mathfrak{\pi}$. Then $\mathcal{R}^{\text {red }} / P_{n, v, \epsilon} \mathcal{R}^{\text {red }}$ is a local factor of a product of fields by the semisimplicity of $\mathcal{R}^{\text {red }}$ and Hida's control theorem. So it is a field and $\mathcal{R}_{P_{\kappa}}^{\text {red }}$ is a regular ring. The primes of $\mathcal{R}$ containing the Hecke operators dividing the tame level thus verify the property required in Theorem 3.7.

The proof of this theorem will be given in §3.7. Before explaining the proof, we need some preliminary results, stated in $\S \S 3.4,3.5$ and 3.6.

3.4. Freeness of $\mathbb{W}$. Recall that we are assuming in this section that $r \leq 1$, so that $\boldsymbol{B}$ is a division algebra. We start by providing a different description of $\mathbb{W}$. Define

$$
\begin{gathered}
\mathcal{V}_{\alpha}:=H^{r}\left(X\left(S\left(p^{\alpha}\right)\right), K / \mathcal{O}\right), \quad \mathcal{V}_{\infty}:=\underset{\alpha}{\lim } \mathcal{V}_{\alpha}, \\
\mathcal{V}_{\alpha}^{*}:=\operatorname{Hom}_{\mathcal{O}}\left(\mathcal{V}_{\alpha}, K / \mathcal{O}\right), \quad \mathcal{V}_{\infty}^{*}:=\operatorname{Hom}_{\mathcal{O}}\left(\mathcal{V}_{\infty}, K / \mathcal{O}\right),
\end{gathered}
$$

where the direct limit is computed with respect to the projection maps $X\left(S\left(p^{\beta}\right)\right) \rightarrow$ $X\left(S\left(p^{\alpha}\right)\right)$ for $\beta \geq \alpha$. The Hecke algebras $h_{k, w}\left(S_{0}\left(p^{\infty}\right), \epsilon, \mathcal{O}\right)$ defined in $\$ 2.3$ can be equivalently introduced as $\underset{\leftarrow}{\lim _{\alpha}} h_{k, w}^{\prime}\left(S_{0}\left(p^{\alpha}\right), \epsilon, \mathcal{O}\right)$, where $h_{k, w}^{\prime}\left(S_{0}\left(p^{\alpha}\right), \epsilon, \mathcal{O}\right)$ is the image in $\operatorname{End}_{\mathcal{O}}\left(H^{r}\left(X\left(S\left(p^{\alpha}\right)\right), \mathscr{L}(n, v, \epsilon, K / \mathcal{O})\right)\right.$ of the algebra generated over 
$\mathcal{O}$ by the Hecke operators; the same observation holds for the ordinary parts: see [8], $\S 3$, for details.

By Lemma 3.4,

$$
\mathbb{W}=H^{r}\left(X(S), \mathcal{D}_{X}\right) \simeq H^{r}\left(X(S), \lim _{\longleftarrow} \mathcal{F}_{\alpha}\right) .
$$

Since

$$
H^{r}\left(X(S), \mathcal{F}_{\alpha}\right)=H^{r}\left(X(S), p_{\alpha *} \mathcal{O}\right) \simeq H^{r}\left(X\left(S\left(p^{\alpha}\right)\right), \mathcal{O}\right),
$$

there is an isomorphism

$$
\mathbb{W} \stackrel{\sim}{\longrightarrow} \underbrace{\lim }_{\alpha} H^{r}\left(X\left(S\left(p^{\alpha}\right)\right), \mathcal{O}\right)
$$

By Poincaré duality:

$$
H^{r}\left(X\left(S\left(p^{\alpha}\right)\right), \mathcal{O}\right) \simeq H_{r}\left(X\left(S\left(p^{\alpha}\right)\right), \mathcal{O}\right)
$$

By Pontryagin duality there is a canonical isomorphism:

$$
\operatorname{Hom}_{\mathcal{O}}\left(H^{r}\left(X\left(S\left(p^{\alpha}\right)\right), K / \mathcal{O}\right), K / \mathcal{O}\right) \simeq H_{r}\left(X\left(S\left(p^{\alpha}\right)\right), \mathcal{O}\right),
$$

where $K:=\operatorname{Frac}(\mathcal{O})$. Since

$$
\mathcal{V}_{\infty}=\underset{\alpha}{\lim } H^{r}\left(X\left(S\left(p^{\alpha}\right)\right), K / \mathcal{O}\right)
$$

there is an isomorphism

$$
\operatorname{Hom}_{\mathcal{O}}\left(\underset{\alpha}{\lim } H^{d}\left(X\left(S\left(p^{\alpha}\right)\right), K / \mathcal{O}\right), K / \mathcal{O}\right) \stackrel{\sim}{\longrightarrow} \mathcal{V}_{\infty}^{*}
$$

By composing the maps (8), (9), (10), (11), we get an isomorphism

$$
\mathbb{W} \stackrel{\sim}{\longrightarrow} \mathcal{V}_{\infty}^{*}
$$

The isomorphism (12) is equivariant for the action of the Hecke operators and, if $r=1$, also for the action of the complex conjugation $\tau$.

Proposition 3.9. The group $\mathbb{W}^{\text {ord }}$ of ordinary $\Lambda$-adic modular symbols is a $\Lambda$-module free of finite rank.

Proof. This follows from Proposition 3.5 and [7], Theorem 3.8, in light of (12). 


\subsection{Description of $\operatorname{Ker}\left(\rho_{\kappa}\right)$}

Proposition 3.10. The kernel of $\rho_{n, v, \epsilon}$ is equal to $P_{n, v, \epsilon} \mathbb{W}^{\text {ord }}$.

Proof. This result is Theorem 5.1 in [2], so only a sketch of the proof will be given.

Recall that a cocycle in $H^{q}\left(\bar{\Gamma}^{\lambda}, \mathbb{D}_{X}\right)$ is represented by the class of an element in $\operatorname{Hom}_{\bar{\Gamma} \lambda}\left(F_{q}, \mathbb{D}_{X}\right)$, where $F_{\bullet}$ is a projective resolution of $\mathbb{Z}$ over $\mathbb{Z}\left[\bar{\Gamma}^{\lambda}\right]$ by right $\bar{\Gamma}^{\lambda}$-modules. Let $\varphi^{(m)}$ denote the characteristic functions of $\pi^{m} Y$, for $m \geq 0$ an integer.

We first prove that $\operatorname{Ker}\left(\rho_{n, v, \epsilon}\right) \supseteq P_{n, v, \epsilon} \mathbb{W}^{\text {ord }}$. Let $\Phi \in P_{n, v, \epsilon} \mathbb{W}^{\text {ord }}$ and write $\Phi=\left(\Phi_{1}, \ldots, \Phi_{h}\right)$. Fix $\lambda$ and represent $\Phi_{\lambda}$ by a cocycle $z$ as above. It follows from [2], Lemma 6.3, that $\int_{X} \varphi^{(m)} d z(f)=0$ for all $f \in F_{d}^{\lambda}$ and all characteristic functions $\varphi^{(m)}$. Since the function

$$
((x, y), z) \longmapsto \epsilon(x) z^{v}(x Y-y X)^{k}
$$

appearing in the specialization map $\rho_{n, v, \epsilon}$ can be written as a uniform limit of functions $\varphi^{(m)}$, the inclusion follows.

Now we show that $\operatorname{Ker}\left(\rho_{n, v, \epsilon}\right) \subseteq P_{n, v, \epsilon} \mathbb{W}^{\text {ord }}$. Let $c \in \operatorname{Hom}_{\bar{\Gamma} \lambda}\left(F_{k}^{\lambda}, \mathbb{D}_{X}\right)$ and choose $b$ such that $c=T\left(p^{m}\right) b$ : this is possible because $T(p)$ induces an isomorphism on $\mathbb{W}^{\text {ord }}$ and, since $p$ is a principal ideal of $\mathfrak{r}$, the $T(p)$ operator preserves each of the cohomology groups $H^{r}\left(\bar{\Gamma}^{\lambda}, \mathbb{D}_{X}\right)$. Set $\pi:=\left(\begin{array}{ll}1 & 0 \\ 0 & p\end{array}\right)$. Write $\Gamma^{\lambda} \pi^{m} \Gamma^{\lambda}=\bigsqcup_{t} \Gamma^{\lambda} \alpha_{\lambda, t}$ and $\gamma_{\lambda, t}:=\pi^{-m} \alpha_{\lambda, t}$. By [2], Lemma 6.1,

$$
\int_{X} \varphi^{(m)}(y) d c(f)(y)=\sum_{t} \int_{X} \varphi^{(m)}\left(y * \alpha_{\lambda, t}\right) d b\left(f \gamma_{\lambda, t}^{-1}\right)(y) .
$$

Since $X_{m} \cap X * \alpha_{\lambda, t}=\emptyset$ for $\alpha_{\lambda, t} \neq 1$ by [2], Lemma 6.6, the above sum is equal to

$$
\int_{X_{m}} d b\left(f \gamma_{\lambda, 1}^{-1}\right)(y)=\rho_{n, v, \epsilon}^{*}\left(\Phi_{\lambda}\right)\left(f \gamma_{\lambda, 1}^{-1}\right)=0 .
$$

From [2], Lemma 6.3, if follows that $b$ takes values in $P_{n, v, \epsilon} \mathbb{D}_{X}$. Hence, $b$ belongs to the image of $H^{r}\left(\bar{\Gamma}^{\lambda}, P_{n, v, \epsilon} \mathbb{D}_{X}\right)$ in $H^{r}\left(\bar{\Gamma}^{\lambda}, D_{X}\right)$ which, by [2], Lemma 1.2, is equal to $P_{n, v, \epsilon} H^{r}\left(\bar{\Gamma}^{\lambda}, \mathbb{D}_{X}\right)$.

3.6. Dimension bounds. We retain the notation and assumptions from previous sections, so $r \leq 1$ and $\boldsymbol{B}$ is a division algebra. Let $P_{\kappa}$ be an arithmetic point such that $\mathcal{R}_{P_{\kappa}}$ is a regular local ring. Let $\mathcal{R}_{P_{\kappa}}$ denote the localization of $\mathcal{R}$ at $P_{\kappa}$. Set $\mathcal{K}_{P_{\kappa}}:=$ $\operatorname{Frac}\left(\mathcal{R}_{P_{\kappa}}\right)$. Let $\mathcal{V}_{\infty}^{*}$,ord denote the ordinary submodule of $\mathcal{V}_{\infty}^{*}$. For any arithmetic character $P_{n, v, \epsilon}$ which factors through $\mathcal{R}_{P_{\kappa}}$, set $\mathcal{V}_{\infty, P_{n, v, \epsilon}}^{* \text { ord }}:=\mathcal{V}_{\infty}^{* \text {,ord }} \otimes_{\Lambda} \mathcal{R}_{P_{n, v, \epsilon}}$.

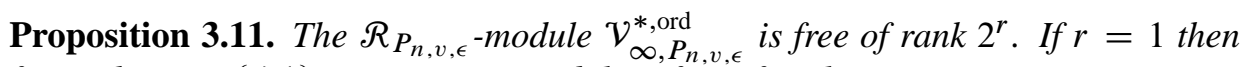
for each $\operatorname{sgn} \in\{ \pm 1\}$, its sgn-eigenmodule is free of rank one. 
Proof. Since $\mathcal{R}_{P_{n, v, \epsilon}}$ is a flat extension of $\Lambda_{P_{n, v, \epsilon}}$ and $\mathcal{V}_{\infty}^{*, \text { ord }}$ is free of finite rank, say $s$, over $\Lambda$, it follows that $\mathcal{V}_{\infty, P_{n, v, \epsilon}^{*} \text {,ord }}$ is free of rank $s$ over $\mathcal{R}_{P_{n, v, \epsilon}}$.

If $r=0$ define $V:=\mathcal{V}_{\infty, P_{n, v, \epsilon}}^{*, \text { ord }}$. If $r=1$ then $\mathcal{V}_{\infty, P_{n, v, \epsilon}^{*}, \text { ord }}$ splits into the direct sum of two submodules

$$
\mathcal{V}_{\infty, P_{n, v, \epsilon}}^{*, \text { ord }}=V_{+} \oplus V_{-}
$$

such that the complex conjugation $\tau$ acts on $V_{ \pm}$as \pm ; in this case, let $V$ denote one of the two modules $V_{ \pm}$. By [7], §4, p. 187, there is an isomorphism

$$
V / P_{n, v, \epsilon} V \stackrel{\sim}{\longrightarrow} h_{k, w}^{\text {ord }}\left(S_{0}\left(p^{\alpha}\right), \epsilon, K\right) .
$$

By (3), $h_{k, w}^{\text {ord }}\left(S_{0}\left(p^{\alpha}\right), \epsilon, K\right)$ is free of rank one over $\mathcal{R}_{P_{n, v, \epsilon}} / P_{n, v, \epsilon} \mathcal{R}_{P_{n, v, \epsilon}}$. Thus

$$
V / P_{n, v, \epsilon} V \simeq \mathcal{R}_{P_{n, v, \epsilon}} / P_{n, v, \epsilon} \mathcal{R}_{P_{n, v, \epsilon}} .
$$

So the $\mathcal{R}_{n, v, \epsilon}$-rank of $V$ is one and the result follows.

Corollary 3.12. The $h_{\kappa}$-eigenspace of $\mathbb{W}_{\mathscr{L}} \otimes \mathscr{L} \mathcal{K}_{P_{\kappa}}$ has dimension at least $2^{r}$ over $\mathcal{K}_{P_{\kappa}}$.

Proof. As in the proof of Proposition 3.11, let $V:=\mathcal{V}_{\infty, P_{n, v, \epsilon}}^{*, \text { if }} r=0$ and $V:=V_{\mathrm{sgn}}$ for $\operatorname{sgn} \in\{ \pm 1\}$ if $r=1$, where $V_{\mathrm{sgn}}$ is defined in (13). Set

$$
V_{\kappa}:=V \otimes_{\mathcal{R}_{P_{n, v, \epsilon}}} \mathcal{R}_{P_{\kappa}} .
$$

We have an Hecke-equivariant map of finite dimensional $\mathcal{K}_{P_{\kappa}}$-vector spaces

$$
\mathbb{W}_{\mathscr{L}} \otimes \mathscr{L}_{\mathcal{L}} \mathcal{K}_{P_{\kappa}} \longrightarrow V_{\kappa} \otimes_{\mathcal{R}_{P_{K}}} \mathcal{K}_{P_{\kappa}} .
$$

If $r=1$, this map is also equivariant for the action of complex conjugation $\tau$. Since $\mathscr{H}$ is commutative if $r=0$ (respectively, $\mathscr{H}[\tau]$ if $r=1$ ) and the $h_{\kappa}$-eigenspace of $V_{\kappa} \otimes_{\mathcal{R}_{P_{K}}} \mathcal{K}_{P_{\kappa}}$ is non-trivial, being of dimension 1 over $\mathcal{K}_{P_{\kappa}}$, it follows that the $h_{\kappa}$-eigenspace (respectively, the $\left(h_{\kappa}\right.$, sgn)-eigenspace) in $\mathbb{W}_{\mathscr{L}} \otimes_{\mathscr{L}} \mathcal{K}_{P_{K}}$ is non-zero too (for this linear algebra argument, see Lemma 5.10 in [15]). This concludes the proof in both cases.

3.7. Proof of the Control Theorem. Before proving Theorem 3.7, recall the following lemma. Let $I_{h}:=\operatorname{Ker}(h)$ be the kernel of the canonical map $h: \mathcal{H} \rightarrow \mathcal{R}$.

Lemma 3.13. Let $M$ be an $\mathscr{H}$-module and $P$ be an ideal in $\Lambda$. Suppose that $P$ is generated by an $M$-regular sequence $\left(x_{1}, \ldots, x_{r}\right)$. Then the image of the map

$$
i_{*}: \operatorname{Ext}_{\mathscr{H}}^{*}\left(\mathscr{H} / I_{h}, P M\right) \longrightarrow \operatorname{Ext}_{\mathscr{H}}^{*}\left(\mathscr{H} / I_{h}, M\right)
$$

induced by the inclusion $i: P M \rightarrow M$ is equal to $P \operatorname{Ext}_{\mathscr{H}}^{*}\left(\mathscr{H} / I_{h}, M\right)$. 
Proof. The proof of this lemma is a slight modification of that of [2], Lemma 1.2, and will be omitted.

Remark 3.14. In order to apply this lemma in the proof of the main theorem, we first note that for any $\mathcal{R}_{P_{\kappa}}$-module $M$ with an $\mathscr{H}$-action, the Hecke eigenspace $M^{h_{\kappa}}$ is equal to $\operatorname{Hom}_{\mathscr{H}}\left(\mathcal{R}_{P_{K}}, M\right)$ and hence to $\operatorname{Ext}_{\mathcal{H}}^{0}(\mathcal{R}, M)$. Since we assume that the generators of $P_{\kappa}$ form a regular sequence in $\mathcal{R}_{P_{K}}$, it follows that they form a regular sequence for $M=\mathbb{W} \otimes \mathcal{R}_{P_{\kappa}}$ since it is free over $\mathcal{R}_{P_{\kappa}}$.

Proof of Theorem 3.7. The proof of the Control Theorem follows [4] and can be described as follows. Recall that we have to show that for each primitive arithmetic point $\kappa$ of weight $(n(\kappa), v(\kappa))$ and character $\epsilon(\kappa)$ the map

$$
\rho_{\kappa}:\left(\mathbb{W} \otimes_{\Lambda} \mathcal{R}_{P_{\kappa}}\right)^{h_{\kappa}} / P_{\kappa}\left(\mathbb{W} \otimes_{\Lambda} \mathcal{R}_{P_{\kappa}}\right)^{h_{\kappa}} \longrightarrow \mathbb{W}_{\kappa}
$$

is an isomorphism.

Since $h_{\kappa}(T(p))$ is a unit in $\mathcal{R}$, the module ( $\left.\mathbb{W} \otimes_{\Lambda} \mathcal{R}_{P_{\kappa}}\right)^{h_{\kappa}}$ is contained in the ordinary part $\mathbb{W}^{\text {ord }} \otimes_{\Lambda} \mathcal{R}_{P_{\kappa}}$. Since, by Proposition 3.10, $\mathbb{W}^{\text {ord }} \otimes_{\Lambda} \Lambda_{P_{\kappa}}$ is a free $\Lambda_{P_{\kappa}}$-module of finite rank, it follows that $\mathbb{W}$ ord $\otimes_{\Lambda} \mathcal{R}_{P_{\kappa}}$ is a free $\mathcal{R}_{P_{\kappa}}$-module of finite rank. By Proposition 3.10 and the fact that $\mathcal{R}_{P_{\kappa}}$ is free over $\Lambda_{P_{\kappa}}$, it follows that the kernel of the map $\mathbb{W}^{\text {ord }} \otimes_{\Lambda} \mathcal{R}_{P_{\kappa}} \rightarrow \mathbb{W}_{0}^{\text {ord }}$ is $P_{\kappa}\left(\mathbb{W} \otimes_{\Lambda} \mathcal{R}_{P_{K}}\right)^{\text {ord }}$. This is the same as $P_{\kappa}\left(\mathbb{W} \otimes_{\Lambda} \mathcal{R}_{P_{\kappa}}\right)^{h_{\kappa}}$ by Lemma 3.13 and the remark following it. Hence, we get an injective map

$$
\left(\mathbb{W} \otimes_{\Lambda} \mathcal{R}_{P_{\kappa}}\right)^{h_{\kappa}} / P_{\kappa}\left(\mathbb{W} \otimes_{\Lambda} \mathcal{R}_{P_{\kappa}}\right)^{h_{\kappa} \hookrightarrow} \mathbb{W}_{\kappa}^{f_{\kappa}} .
$$

Since $\mathbb{W}_{\mathcal{K}}^{f_{K}}$ is $2^{r}$-dimensional, to prove the surjectivity of the map it suffices to show that $\left(\mathbb{W} \otimes_{\Lambda} \mathcal{R}_{P_{K}}\right)^{h_{\kappa}}$ has $\mathcal{R}_{P_{\kappa}}$-rank at least $2^{r}$. Recall that by Corollary 3.12 the $h_{\kappa}$-eigenspace of $\mathbb{W}_{\mathscr{L}} \otimes \mathscr{L} \mathcal{K}_{P_{K}}$ has dimension $2^{r}$ over $\mathcal{K}_{P_{\kappa}}$. The intersection of this eigenspace with $\mathbb{W} \otimes_{\Lambda} \mathcal{R}_{P_{\kappa}}$ is a $\mathcal{R}_{P_{\kappa}}$-submodule of $\left(\mathbb{W} \otimes_{\Lambda} \mathcal{R}_{P_{\kappa}}\right)^{h_{\kappa}}$ of rank $2^{r}$. The surjectivity of the above map follows.

Finally, in the case $r=1$, since the specialization map commutes with the action of the complex conjugations, we deduce the statement on eigenspaces.

\section{References}

[1] A. Ash and G. Stevens, Modular forms in characteristic $l$ and special values of their $L$-functions. Duke Math. J. 53 (1986), no. 3, 849-868. Zbl 0618.10026 MR 0860675

[2] A. Ash and G. Stevens, $p$-adic deformations of cohomology classes of subgroups of GL $(n, \mathbb{Z})$. Collect. Math. 48 (1997), no. 1-2, 1-30. Zbl 0866.20038 MR 1464013

[3] K. Fujiwara, Deformation rings and Hecke algebras in the totally real case. Preprint 2006; arXiv:math/0602606v2 [math.NT]. 
[4] R. Greenberg and G. Stevens, $p$-adic $L$-functions and $p$-adic periods of modular forms. Invent. Math. 111 (1993), no. 2, 407-447. Zbl 0778.11034 MR 1198816

[5] R. Hartshorne, On the de Rham cohomology of algebraic varieties. Inst. Hautes Études Sci. Publ. Math. 45 (1975), 5-99. Zbl 0326.14004 MR 0432647

[6] H. Hida, On $p$-adic Hecke algebras for $\mathrm{GL}_{2}$ over totally real fields. Ann. of Math. (2) 128 (1988), no. 2, 295-384. Zbl 0658.10034 MR 0960949

[7] H. Hida, On nearly ordinary Hecke algebras for GL(2) over totally real fields. In Algebraic number theory, Adv. Stud. Pure Math. 17, Academic Press, Boston, Mass., 1989, 139-169. Zbl 0742.11026 MR 1097614

[8] H. Hida, p-adic ordinary Hecke algebras for GL(2). Ann. Inst. Fourier (Grenoble) 44 (1994), no. 5, 1289-1322. Zbl 0819.11017 MR 1313784

[9] K. Kitagawa, On standard $p$-adic $L$-functions of families of elliptic cusp forms. In $p$ adic monodromy and the Birch and Swinnerton-Dyer conjecture (Boston, Mass., 1991), Contemp. Math. 165, Amer. Math. Soc., Providence, RI, 1994, 81-110. Zbl 0841.11028 MR 1279604

[10] Yu. I. Manin, Non-Archimedean integration and $p$-adic Jacquet-Langlands $L$-functions. Uspehi Mat. Nauk 31 (1976), no. 1 (187), 5-54 (in Russian). Zbl 0336.12007 MR 0417134

[11] Y. Matsushima and G. Shimura, On the cohomology groups attached to certain vector valued differential forms on the product of the upper half planes. Ann. of Math. (2) 78 (1963), 417-449. Zbl 0141.38704 MR 0155340

[12] B. Mazur, J. Tate, and J. Teitelbaum, On $p$-adic analogues of the conjectures of Birch and Swinnerton-Dyer. Invent. Math. 84 (1986), no. 1, 1-48. Zbl 0699.14028 MR 0830037

[13] J. Nekovář, Selmer complexes. Astérisque 310 (2006). Zbl 1211.11120 MR 2333680

[14] A. Panchishkin, Motives over totally real fields and $p$-adic $L$-functions. Ann. Inst. Fourier (Grenoble) 44 (1994), no. 4, 989-1023. Zbl 0808.11034 MR 1306547

[15] R. Pollack and G. Stevens, Explicit computations with overconvergent modular symbols. Preprint, 2004; http://math.bu.edu/people/ghs/research.d/ExplicitOC.pdf

[16] G. Shimura, The special values of the zeta functions associated with Hilbert modular forms. Duke Math. J. 45 (1978), no. 3, 637-679. Zbl 0394.10015 MR 0507462

Received December 20, 2007; revised April 2, 2009

Baskar Balasubramanyam, California Institute of Technology, Pasadena, CA 91125, U.S.A.

E-mail: baskar.balasubramanyam@gmail.com

Matteo Longo, Dipartimento di Matematica Pura e Applicata, Via Trieste 63, 35121 Padova, Italy

E-mail: mlongo@math.unipd.it 